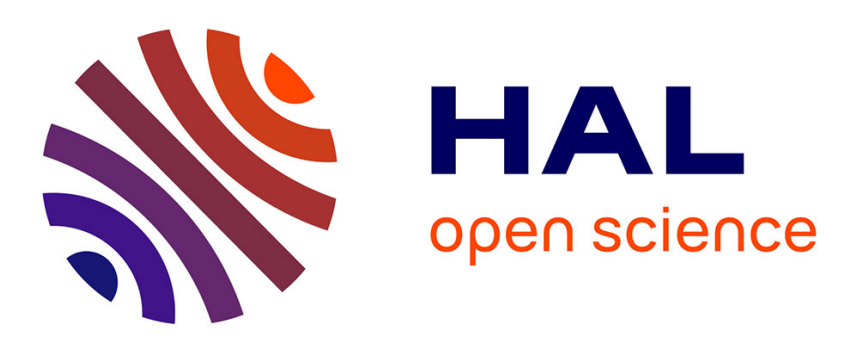

\title{
Evaluation des aménités urbaines par la méthode des prix hédoniques : une application au cas de la ville d'Angers
}

\author{
Muriel Travers, Gildas Appéré, Solène Larue
}

\section{To cite this version:}

Muriel Travers, Gildas Appéré, Solène Larue. Evaluation des aménités urbaines par la méthode des prix hédoniques: une application au cas de la ville d'Angers. Economie et Statistique / Economics and Statistics, 2013, 460-461, pp.145-163. hal-03363731

\section{HAL Id: hal-03363731 \\ https://univ-angers.hal.science/hal-03363731}

Submitted on 4 Oct 2021

HAL is a multi-disciplinary open access archive for the deposit and dissemination of scientific research documents, whether they are published or not. The documents may come from teaching and research institutions in France or abroad, or from public or private research centers.
L'archive ouverte pluridisciplinaire HAL, est destinée au dépôt et à la diffusion de documents scientifiques de niveau recherche, publiés ou non, émanant des établissements d'enseignement et de recherche français ou étrangers, des laboratoires publics ou privés. 


\title{
Évaluation des aménités urbaines par la méthode des prix hédoniques : une application au cas de la ville d'Angers
}

\author{
Muriel Travers *, Gildas Appéré ** et Solène Larue ***
}

\begin{abstract}
La question des choix résidentiels des ménages constitue l'une des problématiques centrales de l'économie urbaine actuelle. Si les premiers modèles monocentriques se focalisaient sur l'arbitrage des ménages entre les coûts de localisation et les coûts de déplacements entre le domicile et le lieu de travail supposé se situer au centre-ville économique, les travaux plus récents cherchent à dépasser ce cadre simplificateur afin de rendre compte des évolutions urbaines actuelles et des différences observées entre les villes européennes et nord-américaines. En particulier, la prise en considération du rôle des aménités urbaines et naturelles améliore le pouvoir explicatif de ces modèles théoriques urbains.
\end{abstract}

Largement utilisée dans le domaine de l'évaluation environnementale, la méthode des prix hédoniques peut apporter des éléments pertinents à cette problématique urbaine. Développée par Rosen (1974), elle permet de mesurer, à partir des comportements des ménages, la valeur que ces derniers accordent aux différentes caractéristiques intrinsèques et extrinsèques des logements, en particulier aux différentes aménités naturelles ou construites. L'estimation de ces prix hédoniques doit tenir compte du double caractère spatial et endogène des caractéristiques des logements : des estimations naïves par moindres carrés ordinaires sont potentiellement biaisées. Corriger ces biais requiert l'utilisation de la méthode FGS2SLS proposée par Fingleton et Le Gallo (2008), combinant variables instrumentales et correction de l'autocorrélation spatiale.

Rappel :

Les jugements et opinions exprimés par les auteurs n'engagent qu'eux mêmes, et non les institutions auxquelles ils appartiennent, ni a fortiori l'Insee.
Cette méthode est mise en œuvre dans le cas de la ville d'Angers : avec un centre bien pourvu en aménités historiques, il est en effet typique du cas européen. Au-delà du rôle prépondérant des caractéristiques intrinsèques des logements, la valorisation par les ménages des aménités « vertes » telles que les espaces verts souligne la complexité de l'espace urbain angevin.

\footnotetext{
* UMR GRANEM, 13, allée François Mitterrand, BP 3633, 49036 Angers cedex 01, muriel.travers@univ-angers.fr.

** UMR GRANEM, 13, allée François Mitterrand, BP 3633, 49036 Angers cedex 01.

*** Emprou s.a.r.l., 18 rue Pierre Capésius, L-5814 Fentange, Luxembourg.

Les auteurs remercient Johanna Choumert pour sa contribution aux premières étapes de ce travail. Ils remercient également Julie Le Gallo ainsi que les référés pour leurs très utiles commentaires.
} 
$\grave{\mathbf{A}}$ la suite du mouvement de décentralisation amorcé au début des années 1980 par les lois Defferre opérant un transfert de compétences de l'État central vers les collectivités territoriales, l'aménagement du territoire et l'amélioration du cadre de vie local sont désormais de la responsabilité des décideurs locaux. L'offre d'une gamme étendue de services publics et, plus généralement, d'aménités urbaines constitue alors un outil majeur de développement local. Dans un tel contexte, la connaissance des préférences des ménages concernant les aménités urbaines s'impose comme un élément important du processus de décision publique concernant l'aménagement des zones urbaines.

L'objet de cet article est de montrer que la méthode des prix hédoniques permet de révéler les préférences des ménages pour de telles aménités. Cependant, le recours à la méthode des prix hédoniques stricto sensu (i.e. non couplés aux salaires hédoniques) dans une problématique spécifiquement urbaine nécessite que l'on restreigne l'analyse à des aménités dont l'influence se limite aux choix intra-urbains et que l'on dispose d'une caractérisation géographique suffisamment précise des données. Il requiert également la mise en œuvre de modèles d'économétrie spatiale afin de prendre en compte une éventuelle autocorrélation spatiale. Nous appliquons cette méthode au cas de la ville d'Angers et montrons que les ménages attribuent effectivement une valeur significative aux espaces verts.

\section{La méthode des prix hédoniques permet d'évaluer les aménités urbaines}

D u point de vue de l'économie urbaine néoclassique (Alonso, 1964 ; Muth, 1969), l'existence d'une ville se justifie au travers de sa capacité à réduire les distances entre les agents économiques : la proximité entre les agents conjuguée à une forte densité de population est, en effet, un facteur d'efficacité économique car elle permet de minimiser les coûts et les temps de transport et de mise en contact. La ville résulte ainsi des décisions de localisation des firmes, des ménages et des administrations publiques. Dans cet article, nous nous intéressons spécifiquement aux déterminants des choix résidentiels des ménages.

\section{Les aménités jouent un rôle important dans les choix résidentiels...}

Le modèle urbain néoclassique proposé par Alonso (1964), puis étendu par Muth (1969) et Mills $(1967,1972)$ prend comme cadre de référence le cas de nombreuses villes nordaméricaines, à savoir, schématiquement, des villes monocentriques dont les emplois sont situés au centre-ville (Central Business District), où les individus ne se rendent que pour travailler. Cette modélisation cherche ainsi à tenir compte de l'arbitrage que font les ménages entre les coûts de localisation et les coûts de mobilité associés aux déplacements entre le domicile et le lieu de travail situé au centre-ville. Dans ce modèle, toute augmentation du prix unitaire de la variable de logement implique un rapprochement du centre afin de bénéficier d'une baisse du coût de transport. Le succès du modèle monocentrique vient du fait qu'il parvient, à partir de développements relativement simples, à rendre compte de certaines régularités observées empiriquement concernant la répartition spatiale des populations, des activités économiques et des valeurs foncières. Cependant, cette modélisation a été critiquée du fait justement de son extrême simplicité et de sa difficulté à appréhender la diversité et l'évolution de la réalité urbaine : si elle a pu rendre compte de manière satisfaisante de la situation des villes américaines, caractérisées par la concentration des ménages pauvres au centre-ville et par la localisation des ménages riches dans les banlieues, elle est moins adaptée aux grandes villes européennes, où la situation inverse prédomine.

Le dépassement du modèle monocentrique dans lequel l'emploi situé au centre-ville conditionne le choix résidentiel des ménages, amène logiquement à s'interroger sur les autres facteurs influençant ces choix. L'introduction du rôle des aménités urbaines permet d'améliorer la compréhension du choix résidentiel des ménages. Paradoxalement, le concept d'aménité, alors qu'il est couramment utilisé dans les travaux d'économie environnementale ou urbaine, est rarement défini de manière précise. Il recouvre, selon les sources, une gamme extrêmement diverse d'éléments tels que les forêts, la qualité de l'air, des services publics de qualité, les espaces verts, les monuments historiques mais aussi la présence de restaurants (Brueckner et al., 1999), la sécurité (Blomquist et al., 1988) ou le charme d'un quartier (Bartik et Smith, 1987). Dans cet article, nous nous inspirons de la définition proposée par Bartik et Smith (1987), à savoir qu'une aménité (resp. désaménité) 
est une caractéristique géographiquement localisée agissant de manière positive (resp. négative) sur les agents économiques. Une telle définition est suffisamment large pour englober la diversité des éléments évoqués ci-dessus. Elle permet en outre de souligner l'importance de la localisation et du voisinage et, par-delà, le fait que l'influence de l'aménité ${ }^{1}$ décroît avec la distance et/ou l'accessibilité à cette dernière.

Cependant, quelle que soit la définition donnée à la notion d'aménités, leur prise en compte permet de rendre les modèles d'économie urbaine plus réalistes en dépassant l'arbitrage initial entre l'accès au centre-ville économique (Central Business District) et la surface de logement désirée (ou toute autre variable caractérisant la quantité consommée de service de logement). Ainsi, la présence d'aménités non localisées dans le centre-ville et recherchées par les ménages telles que les forêts ou la proximité $\mathrm{du}$ littoral permet de contrebalancer la force d'attraction du centre-ville (Straszheim, 1987) et d'expliquer, dans une certaine mesure, le phénomène actuel d'étalement urbain.

D'un point de vue empirique, chercher à mesurer la valeur que les ménages accordent à une aménité donnée permet d'évaluer son importance dans la décision des ménages. La méthode des prix hédoniques est l'une des principales méthodes permettant d'estimer de telles valeurs.

\section{... et il est possible de les évaluer au moyen de la méthode des prix hédoniques}

La méthode des prix hédoniques (cf. encadré 1) a connu un développement important dans le domaine de l'économie de l'environnement du fait de sa capacité à mesurer à partir des comportements observés les valeurs que les ménages attribuent à diverses aménités naturelles. Dans une perspective d'économie urbaine, cette méthode contribue à une meilleure compréhension des choix résidentiels des ménages, non seulement en prenant explicitement en compte la complexité des biens immobiliers mais également en mesurant l'influence possible des différentes aménités environnementales sur les choix résidentiels de ces ménages, et ce, au travers de la mesure de la valeur qu'ils attribuent à ces aménités.

1. Influence mesurable in fine au travers de son impact sur une variable d'intérêt telle que la densité de la population ou le gradient de prix des logements.

Encadré 1

\section{LES PRINCIPES DE LA MÉTHODE DES PRIX HÉDONIQUES}

La méthode des prix hédoniques (Rosen, 1974) part du principe qu'un bien immobilier est un bien hétérogène composé d'un ensemble de caractéristiques $Z=\left(z_{1}, \ldots, z_{k}, \ldots, z_{k}\right)$ vendues en bloc. En effet, les résidences se distinguent les unes des autres, à la fois au travers de leurs caractéristiques intrinsèques (la surface habitable, le nombre de pièces, l'année de construction, etc.) mais également au travers de leurs caractéristiques extrinsèques, c'est-à-dire au travers d'une localisation différenciée notamment en termes d'aménités et désaménités environnantes. La méthode des prix hédoniques a alors pour objet de révéler, dans une première étape, les prix marginaux implicites de ces différentes caractéristiques à partir du prix global $\mathrm{P}(\mathrm{Z})$ du bien immobilier. À l'équilibre, chaque prix marginal implicite $p_{k}$ est égal au consentement à payer marginal du ménage pour cette caractéristique et est calculé dans le cas de variables quantitatives comme la dérivée du prix global $P(Z)$ par rapport à la quantité $Z_{k}$.

Le calcul empirique des différents prix marginaux implicites nécessite donc d'estimer la fonction de prix hédoniques $\hat{P}(Z)$ en régressant les prix de transaction des logements sur les différentes caractéristiques intrinsèques et extrinsèques de ces derniers. Si on dispose, en outre, des caractéristiques des ménages acheteurs, il est alors possible (sous certaines hypothèses concernant l'offre de caractéristiques), de calculer dans une seconde étape, la valeur que les ménages associent à une variation non marginale d'une caractéristique donnée.

En outre, par la nature même du phénomène étudié, il est nécessaire de prendre en compte dans la modélisation économétrique la relation à double sens entre prix et caractéristiques du bien immobilier : la relation d'intérêt est l'impact des caractéristiques sur le prix mais, en sens, inverse, le prix détermine les caractéristiques des biens immobiliers qui sont achetés. Par exemple, les prix plus élevés conduisent à acheter des logements de plus petite taille. II est nécessaire de tester cet impact du prix sur les différentes caractéristiques de l'habitation lors de l'estimation de la fonction de prix hédoniques. Si les caractéristiques des logements sont endogènes, l'estimation par moindres carrés ordinaires de leur impact sur les prix donnera des résultats biaisés et non convergents, la (ou les) variable(s) explicative(s) $z_{k}$ étant corrélée(s) aux résidus de la régression (Cavailhès, 2005 ; Travers et al., 2008 ; Travers, 2007). II convient alors d'utiliser la méthode des doubles moindres carrés. 
Le recours à la méthode des prix hédoniques stricto sensu pour mesurer la valeur que les ménages attribuent à une aménité donnée n'est pleinement pertinent que dans l'hypothèse où cette aménité n'engendre pas, à elle seule, des choix interurbains de localisation géographique impliquant d'éventuelles variations de salaires. Le cas échéant, il conviendrait alors de calculer le prix implicite complet d'une aménité défini comme la somme du différentiel de prix immobilier et du différentiel (de signe contraire) de salaire, ce qui suppose la mise en œuvre conjointe de la méthode des prix hédoniques et des salaires hédoniques (Rosen (1979), Roback $(1982)^{2}$ et Blomquist et al., (1988)).

\section{Un système d'information géographique permet de positionner les aménités par rapport aux biens immobiliers...}

Par ailleurs, le succès de la mise en œuvre de la méthode des prix hédoniques repose avant tout sur la qualité des données : de fait, la portée de l'analyse est hypothéquée si les caractéristiques des biens immobiliers échangés sont mal renseignées et ce, quelle que soit la sophistication des traitements économétriques utilisés (Sheppard, 1987 ; Cavailhès, 2005). Or, dans le cadre d'une problématique cherchant à mesurer l'influence des aménités environnantes, la connaissance du positionnement de ces dernières revêt un caractère crucial. Qui plus est, à l'échelle de choix résidentiels intra-urbains pour lesquels une distance de quelques centaines de mètres peut radicalement changer les caractéristiques extrinsèques d'une habitation, il convient de situer chaque observation le plus finement possible dans l'espace. Cette caractérisation spatiale devient possible lorsque l'on dispose des données cadastrales des biens immobiliers. À partir de ces dernières, on peut alors mettre en place un système d'information géographique (SIG) positionnant chaque bien immobilier ${ }^{3}$ par rapport aux différents éléments extrinsèques susceptibles d'intervenir dans le choix résidentiel des ménages.

\section{...à condition de tester la présence d'une éventuelle autocorrélation spatiale}

Cette caractérisation géographique d'un bien par rapport à son milieu environnant confère ainsi une dimension spatiale aux données utilisées. Or, dans le cadre d'une analyse intra-urbaine où les observations correspondent par nature à des biens immobiliers proches les uns des autres, l'existence d'une autocorrélation spatiale ${ }^{4}$, correspondant à une absence d'indépendance entre les observations géographiques, semble difficile à exclure a priori. Elle peut avoir pour origine l'existence d'un lien entre le prix d'un logement et le prix observé des autres logements se situant dans son voisinage (adjacency effect, Can, 1992) et/ou l'existence de variables omises présentant une configuration spatiale (Le Gallo, 2002). Ne pas prendre en compte une telle autocorrélation dans les estimations conduit à des valeurs biaisées et non convergentes des paramètres de la fonction de prix hédoniques. Il est donc nécessaire d'en tester la présence. Notons que ces tests devront prendre en compte l'existence d'une possible endogénéité des variables, inhérente à la méthode des prix hédoniques ${ }^{5}$ (cf. encadré 1).

\section{Influence des aménités urbaines sur le choix résidentiel des ménages angevins}

Tous cherchons à mettre en évidence l'influence des aménités urbaines sur le choix résidentiel des ménages dans le cas de la ville d'Angers. Le choix de cette ville nous paraît adapté à cette démarche pour différentes raisons. D'une part, Angers est typique des villes françaises (et plus généralement européennes) de taille moyenne : en raison de son passé (ancienne capitale de l'Anjou) et de sa situation administrative actuelle (préfecture du Maine et Loire), elle concentre de nombreuses aménités historiques en centre-ville. D'autre part, elle détient également un ensemble d'éléments à l'origine d'aménités « modernes » (ex : théâtres, restaurants) ou " vertes », tels que des parcs disséminés sur l'ensemble de la ville et des forêts situées à la périphérie. En raison de cette structure relativement complexe, la ville d'Angers constitue un objet d'étude particulièrement approprié.

2. Ces deux auteurs analysent comment les salaires et les prix immobiliers s'ajustent à des différences d'aménités entre les aires urbaines. Ils calculent également un indice de qualité de vie pour les ménages et de qualité de l'environnement économique pour les entreprises.

3. Via l'utilisation de logiciels dédiés aux systèmes d'information géographique tels que le logiciel ArcGIS.

4. Un second effet, en présence de données spatialisées, peut apparaître, à savoir l'existence d'une hétérogénéité spapeut apparaitre, à savoir lexistence d'une hétérogéneité spacaractéristiques des logements selon leur localisation géographique (Bono et al. ; 2007 ; Le Gallo, 2004).

5. Ces tests sont présentés lors de l'estimation de la fonction de prix hédoniques appliquée à la ville d'Angers. 


\section{Les données}

Les données que nous avons utilisées portent sur 1016 appartements vendus ${ }^{6}$ sur la période 2004-2005. Les prix de vente hors frais d'agence et de notaire ${ }^{7}$ ainsi que les différentes caractéristiques intrinsèques des appartements (nombre de pièces, dotation d'au moins deux salles de bain, étage et période de construction) proviennent de données notariales (PERVAL - Notaires de France) (cf. tableau 1).

L'environnement social, démographique et économique dans lequel se situe le logement constitue également un ensemble de caractéristiques extrinsèques susceptibles d'influencer à la hausse ou à la baisse les prix de vente. Dans la lignée des travaux de Baumont (2009) et de Baumont et Legros (2009), nous avons défini à partir des données géo-localisées de la base PERVAL et des données IRIS de l'Insee ${ }^{8}$, les principales caractéristiques sociodémographiques et économiques de l'IRIS dans lequel se situe l'appartement ${ }^{9}$ (cf. tableau 2).

Ces différents taux, hormis celui concernant les entreprises et établissements de l'IRIS, ont été introduits sous forme de ratio ${ }^{10}$ afin de savoir si le phénomène étudié est surreprésenté ou non dans l'IRIS considéré relativement à la ville d'Angers ${ }^{11}$.

En vue de déterminer le rôle joué par les externalités liées aux paysages, nous avons défini plusieurs indicateurs paysagers. Ainsi, afin d'analyser de manière fine l'influence des espaces verts sur les préférences des ménages, nous avons choisi de calculer la distance ${ }^{12}$ entre l'appartement et l'espace vert le plus proche ${ }^{13}$ mais également un indicateur de superficie de ce dernier. Pour également tenir compte d'un éventuel impact de la densité des espaces verts

6. Les appartements représentent $74,8 \%$ du marché immobilier de la ville d'Angers.

7. Afin de prendre en compte la hausse des prix des appartements observée au cours de l'année 2005 sur le marché immobilier du département du Maine-et-Loire, nous avons déflaté ces derniers (+ 10,7\% /an, soit une hausse équivalente mensuelle de $0,85 \%)$.

8. L'IRIS (Îlot Regroupé pour l'Information Statistique) est un outil développé par l'Insee et se définit comme " un découpage du territoire en mailles de taille homogène "(Insee, 2010). L'Insee en 2008) des IRIS d'activité. Les IRIS d'habitat correspondent à des zones dont la population "se situe en général entre 1800 et 5000 habitants" et sont homogènes en termes d'habitat. Leurs limites s'appuient sur les grandes coupures du tissu urbain (voies principales, voies ferrées, cours d'eau, etc.). Les IRIS d'activité regroupent, quant à eux, plus de 1000 salariés.

9. L'échantillon étudié couvre 49 IRIS sur les 69 existants pour la ville d'Angers soit une couverture de $71 \%$ (la carte des IRIS la ville d'Angers soit une couverture
d'Angers est proposée en annexe 1).

10. Le ratio de la caractéristique $j$ est défini de la manière suivante : taux de la caractéristique $j$ dans I'IRIS i / taux de la caractéristique $j$ dans l'ensemble des IRIS.

11. Une alternative à l'introduction des caractéristiques socioéconomiques dans la fonction de prix hédoniques aurait été d'introduire des indicatrices de quartiers. Cependant, ces derd'introduire des indicatrices de quartiers. Cependant, ces der-
niers regroupant plusieurs caractéristiques intrinsèques et extrinniers regroupant plusieurs caractéristiques intrinsèques et extrinmodèle, limitant ainsi sa portée interprétative. En effet, le quartier du centre-ville d'Angers est celui ayant un taux d'appartements et d'entreprises le plus important. De plus, les appartements de ce quartier sont les plus anciens (construits avant 1947). Le quartier La Fayette Eblé (quartier aisé d'Angers) se distingue quant à tier La Fayette Eblé (quartier aisé d'Angers) se distingue quant à
lui par une diversité en termes d'occupation du sol plus élevée. 12. Les distances ont été calculées à partir de la localisation de chaque appartement et du plan d'occupation du sol de la ville d'Angers (2006) mis à disposition par l'Agence de l'Urbanisme de la Région Angevine (AURA).

13. Les espaces verts angevins ne sont pas concentrés dans une zone géographique particulière, mais répartis sur l'ensemble de la ville d'Angers.

Tableau 1

Descriptif des caractéristiques des appartements

\begin{tabular}{|l|l|}
\hline \multicolumn{1}{|c|}{ Variables } & \multicolumn{1}{c|}{ Description } \\
\hline Prix_ttc & Prix de vente de l'habitation hors frais d'agence et de notaire (en $€$ ) \\
\hline Nbr_piece & Nombre de pièces à vivre de l'appartement (à l'exception des pièces dites « humides » : salle de bain, cuisine, WC) \\
\hline Sdb2_plus & $\begin{array}{l}\text { Présence d'une ou plusieurs salles de bain (avec Sdb2_plus =1 si l'appartement est équipé d'au moins } \\
\text { deux salles de bain (ou salles d'eau ou salles de douche), = 0 sinon) }\end{array}$ \\
\hline Étage & Étage où se situe l'appartement \\
\hline Anc_av_1850 & $=1$ si l'appartement est construit avant 1850, 0 sinon \\
\hline Anc_1850_1913 & $=1$ si l'appartement est construit entre 1850 et 1913, 0 sinon \\
\hline Anc_1914_1947 & $=1$ si l'appartement est construit entre 1914 et 1947, 0 sinon \\
\hline Anc_1948_1969 & $=1$ si l'appartement est construit entre 1948 et 1969, 0 sinon \\
\hline Anc_1970_1980 & $=1$ si l'appartement est construit entre 1970 et 1980, 0 sinon \\
\hline Anc_1981_1991 & $=1$ si l'appartement est construit entre 1981 et 1991, 0 sinon \\
\hline Anc_ap_1992 & $=1$ si l'appartement est construit après 1992,0 sinon \\
\hline
\end{tabular}

Lecture : ce tableau présente le nom, la définition et la nature de chacune des caractéristiques intrinsèques d'un appartement.

Champ : échantillon de 1016 appartements vendus dans la ville d'Angers en 2004 et 2005.

Source : PERVAL - Notaires de France. 
sur les choix résidentiels, nous avons calculé cette dernière pour un rayon de 1000 mètres autour de chaque appartement ${ }^{14}$. Ces différents indicateurs ont été complétés par d'autres indicateurs paysagers, à savoir la distance des appartements à la forêt, à la parcelle de vignoble et à la lande les plus proches.

De même, afin de caractériser de manière plus générale, la composition de l'environnement autour de l'appartement en termes d'occupation du sol, nous avons calculé l'indice de Shannon et le niveau d'agrégation des différents types d'occupation du sol. Rappelons que l'indice de Shannon mesure la distribution des différents types d'occupation du sol à proximité des appartements ainsi que leur plus ou moins grande diversité. Concernant les distances de voisinage retenues pour ces deux indicateurs, nous avons choisi les distances de 100, 500 et 1000 mètres et n'avons retenu pour chaque indicateur que celle donnant les meilleurs résultats en termes d'estimation, à savoir 100 mètres pour l'indice de Shannon et 500 mètres pour l'indicateur d'agrégation.

Ces indicateurs sont définis dans le tableau 3. Les formules utilisées pour le calcul de certains d'entre eux sont présentées en annexe 2.
En complément à ces indicateurs, nous avons calculé la distance euclidienne entre les appartements et le centre-ville d'Angers (cf. tableau 4). Nous devrions constater, toutes choses égales par ailleurs, une baisse des prix des appartements lorsque la distance augmente du fait notamment d'une plus faible accessibilité des ménages angevins aux commerces et aux externalités historiques.

Afin de prendre en compte d'éventuels nuisances ou désagréments liés à la proximité du logement par rapport à un cimetière ${ }^{15}$ ou à des axes routiers ${ }^{16}$, nous avons également mesuré la distance entre les appartements et ces deux types

14. Les distances de 100 et 500 mètres ont été également testées. Néanmoins, le modèle utilisant la distance de 1000 mètres aboutissant à de meilleurs résultats en termes d'estimation, distance de 1000 mètres a été conservée lors de l'estimation de la fonction de prix hédoniques.

15. Cette caractéristique a été également introduite dans l'analyse hédonique réalisée par Anderson et West (2006) et par Klaiber et Phaneuf (2010).

16. Nous avons choisi d'utiliser la distance de l'appartement à ces infrastructures routières afin de tenir compte du bruit occasionné par ces dernières. Kim et al. (2007) utilisent également cette distance et montrent que la proximité des logements de Sécul ents de Seoul (Corée) aux voies principales a eu un impact négatif et significatif sur les valeurs des propriétés vendues entre 2002 et 2004. Sander et al. (2010) observent aussi cette influence négative des routes passantes sur le prix des logements vendus en 2005 dans les Comtés du Dakota et de Ramsey (Minnesota, États Unis).

\section{Tableau 2}

Descriptif des principales caractéristiques socio-économiques des IRIS

\begin{tabular}{|c|c|}
\hline Variables & Description \\
\hline Taux_chômage_15_24ans & Part de la population active de l'IRIS âgée entre 15 et 24 ans étant au chômage \\
\hline Taux_chômage_15_64ans & Part de la population active de l'IRIS âgée de 15 et 64 ans étant au chômage \\
\hline Taux_ACC_15_64ans & $\begin{array}{l}\text { Part de la population active de l'IRIS, âgée de } 15 \text { à } 64 \text { ans, appartenant à la catégorie } \\
\text { "Artisans, commerçants, chefs d'entreprise " }\end{array}$ \\
\hline Taux_CPIS_15_64ans & $\begin{array}{l}\text { Part de la population active de l'IRIS, âgée de } 15 \text { à } 64 \text { ans, appartenant à la catégorie } \\
\text { "Cadres, professions intellectuelles supérieures " }\end{array}$ \\
\hline Taux_PI_15_64ans & $\begin{array}{l}\text { Part de la population active de l'IRIS, âgée de } 15 \text { à } 64 \text { ans, appartenant à la catégorie } \\
\text { "Professions intermédiaires" }\end{array}$ \\
\hline Taux_Emp_15_64ans & $\begin{array}{l}\text { Part de la population active de l'IRIS, âgée de } 15 \text { à } 64 \text { ans, appartenant à la catégorie } \\
\text { «Employés » }\end{array}$ \\
\hline Taux_Ouv_15_64ans & $\begin{array}{l}\text { Part de la population active de l'IRIS, âgée de } 15 \text { à } 64 \text { ans, appartenant à la catégorie } \\
\text { "Ouvriers" }\end{array}$ \\
\hline Taux_0_17 ans & Part de la population de l'IRIS ayant moins de 17 ans \\
\hline Taux_Plus_65_ans & Part de la population de l'IRIS âgée de plus de 65 ans \\
\hline Taux_Famille_3enfants_ou_plus & Part des ménages de I'IRIS ayant trois enfants ou plus âgés de moins 25 ans \\
\hline Taux_Population_Immigrée & Part de la population de l'IRIS étant d'origine immigrée \\
\hline Taux_Appartement & Part des appartements dans I'IRIS considéré \\
\hline Taux_d'HLM & Part des logements de type HLM dans I'IRIS considéré \\
\hline Taux_Logement_Vacant & Part des logements vacants dans I'IRIS considéré \\
\hline Taux_Entreprise_Etablissement & $\begin{array}{l}\text { Nombre d'entreprises et d'établissements de l'IRIS rapporté au nombre total d'entreprises } \\
\text { et d'établissements de la ville d'Angers }\end{array}$ \\
\hline
\end{tabular}

Lecture : ce tableau présente le nom, la définition et la nature de chacune des caractéristiques socio-économiques des IRIS dans lequel se situe l'appartement.

Champ : échantillon de 1016 appartements vendus dans la ville d'Angers en 2004 et 2005.

Source : Insee, Recensement de la population 2006 et Répertoire des entreprises et établissements (SIRENE, 2008). 
d'occupation du sol. Nous avons également intégré dans notre fonction de prix hédoniques la distance des appartements aux installations de santé. L'effet escompté sur le prix de vente est a priori incertain, du fait notamment de l'existence de nuisances sonores occasionnées par les véhicules s'y rendant et pouvant ainsi contrebalancer l'effet positif lié notamment à un accès plus rapide à des soins d'urgence. L'étude menée par Kim et al. (2003) sur le marché immobilier de Séoul (Corée) semble néanmoins indiquer que la proximité du logement par rapport aux établissements de santé peut être perçue comme une aménité par les ménages.

Les valeurs moyennes ${ }^{17}$ des différentes caractéristiques utilisées pour l'estimation de la fonction de prix hédoniques sont présentées en annexe 3 .

\section{Une méthode d'estimation de la fonction de prix hédoniques tenant compte de l'endogénéité et de l'autocorrélation spatiale (méthode FGS2SLS)}

L'estimation de modèles hédoniques est souvent biaisée par la présence d'endogénéité : en effet, les caractéristiques mêmes d'un bien sont souvent co-déterminées avec son prix. De plus,

17. Concernant les caractéristiques socio-économiques de I'IRIS dans lequel se situe l'appartement, ces dernières sont calculées au niveau de la ville d'Angers.

Tableau 3

Définition des indicateurs des aménités « vertes " et d'occupation du sol

\begin{tabular}{|l|l|}
\hline \multicolumn{1}{|c|}{ Indicateur } & \multicolumn{1}{c|}{ Définition } \\
\hline Distance_Lande & $\begin{array}{l}\text { Distance euclidienne entre l'appartement et la lande la plus proche (en mètres). Est considérée } \\
\text { comme lande toute formation végétale basse contenant des surfaces de broussailles alternant } \\
\text { avec des pelouses }\end{array}$ \\
\hline Distance_Vigne & Distance euclidienne entre l'appartement et la parcelle de vignobles la plus proche (en mètres) \\
\hline Distance_Forêt & $\begin{array}{l}\text { Distance euclidienne entre l'appartement et la forêt la plus proche (en mètres). Est considéré comme } \\
\text { forêt tout type de boisements (feuillus, conifères et peuplements mixtes) autre que les peupleraies }\end{array}$ \\
\hline Distance_Esp_vert & $\begin{array}{l}\text { Distance euclidienne entre l'appartement et l'espace vert le plus proche (en mètres). Un espace vert } \\
\text { correspond à un parc ou un jardin public }\end{array}$ \\
\hline Superficie_Esp_vert & Superficie de l'espace vert le plus proche (en m²) \\
\hline Densité_Esp_vert_1000m & $\begin{array}{l}\text { Indicateur de la densité des espaces verts dans un rayon de 1 000 mètres autour de chaque } \\
\text { appartement }\end{array}$ \\
\hline Agrégation_500m & $\begin{array}{l}\text { Indique si, dans un rayon de 500 mètres autour de chaque appartement, les différents types } \\
\text { d'occupation du sol sont agrégés ou répartis en mosaïque (\%). } \\
\text { Si ce coefficient a une valeur élevée, les différents types d'occupation du sol sont agrégés. } \\
\text { Inversement, si ce coefficient a une valeur faible, les différents types d'occupation du sol sont répartis } \\
\text { par petites touches sur l'ensemble de la zone définie }\end{array}$ \\
\hline Shannon_100m & $\begin{array}{l}\text { Cet indicateur indique si l'occupation du sol est concentrée en peu de catégories ou distribuée } \\
\text { de manière équitable entre ces dernières dans un rayon de 100 mètres autour de chaque appartement. } \\
\text { Si cet indicateur a une valeur élevée, il existe alors une diversité importante d'occupation du sol } \\
\text { dans la zone définie autour de l'appartement }\end{array}$ \\
\hline
\end{tabular}

Lecture : ce tableau présente le nom, la définition et l'interprétation des indicateurs paysagers et de composition de l'environnement autour des appartements en termes d'occupation du sol.

Champ : échantillon de 1016 appartements vendus dans la ville d'Angers en 2004 et 2005

Source : d'après l'Agence de l'Urbanisme de la Région Angevine (2006), et d'après les logiciels FRAGSTATS et ArcGIS.

Tableau 4

Définition des indicateurs d'éléments urbains complémentaires pouvant exercer un rôle d'aménité ou de désaménité

\begin{tabular}{|l|l|}
\hline \multicolumn{1}{|c|}{ Indicateur } & \multicolumn{1}{c|}{ Définition } \\
\hline Distance_Centre_ville & Distance euclidienne entre les appartements et le centre-ville d'Angers (Place du Ralliement) \\
\hline Distance_Cimetière & Distance euclidienne en mètres entre l'appartement et le cimetière le plus proche \\
\hline Distance_Axes_routiers & $\begin{array}{l}\text { Distance euclidienne en mètres entre l'appartement et l'infrastructure routière la plus proche, sachant } \\
\text { que les infrastructures routières correspondent à l'ensemble des autoroutes, rocades et voies rapides } \\
\text { d'une largeur minimale d'environ 25 mètres }\end{array}$ \\
\hline Distance_Santé & $\begin{array}{l}\text { Distance euclidienne en mètres entre l'appartement et toutes emprises hospitalières ou liées à la santé } \\
\text { identifiables, maisons de retraite et casernes de pompiers }\end{array}$ \\
\hline
\end{tabular}

Lecture : ce tableau présente le nom et la définition de différents indicateurs urbains susceptibles d'influencer les prix immobiliers.

Champ : échantillon de 1016 appartements vendus dans la ville d'Angers en 2004 et 2005.

Source : d'après le logiciel ArcGIS. 
cette endogénéité est souvent associée à l'autocorrélation spatiale qui apparaît lors de la géolocalisation d'un bien. Ainsi, l'association de ces deux facteurs de biais suppose un traitement particulier de notre modèle.

L'introduction simultanée de l'endogénéité et de l'autocorrélation spatiale est réalisée en règle générale au moyen d'un décalage spatial d'ordre 1 de la variable dépendante. Le décalage spatial permet de tester une influence possible du prix des transactions voisines sur le prix de l'habitation considérée. Afin de prendre en compte d'une part, d'éventuelles variables endogènes autres que le décalage spatial et d'autre part, un processus d'autocorrélation dans les erreurs, Fingleton et Le Gallo (2008) ont étendu la procédure GS2SLS (Generalized Spatial Two-Stage Least Squares) de Kelejian et Prucha $(1998,1999)$. Ils proposent une estimation en trois étapes, le FGS2SLS (Feasible Generalized Spatial Two-Stage Least Squares). Il s'agit d'une méthode combinant à la fois la méthode des variables instrumentales pour prendre en compte l'autocorrélation spatiale (dont la forme est spécifiée) de la variable expliquée et des variables endogènes et la méthode des moments généralisés pour corriger l'autocorrélation spatiale des erreurs. La présentation détaillée et la mise en œuvre de cette méthode au cas angevin sont données dans l'encadré 2 .

Nous avons testé notre modèle grâce à plusieurs méthodes économétriques, de façon à contrôler et à confirmer la présence de biais d'estimation. L'estimation du modèle par moindres carrés ordinaires est présentée afin de montrer les variations qu'il peut exister lors de la correction des différents biais d'estimation. Cependant, l'intégration d'un décalage spatial induit automatiquement la présence d'endogénéité et donc une estimation par une méthode pouvant corriger ce biais, à savoir la méthode des variables instrumentales (IV-SAR). Enfin, cette étape est nécessaire afin de démontrer la présence d'autocorrélation spatiale qui doit être ensuite corrigée par la méthode FGS2SLS. Les différents modèles estimés sont présentés dans le tableau 5 .

L'application du test de Pagan-Hall (1983) au modèle IV-SAR conduit à accepter l'hypothèse nulle d'homoscédasticité des erreurs dans le cadre de variables endogènes. Afin de vérifier la fiabilité des instruments utilisés dans nos estimations en doubles moindres carrés, nous avons appliqué le test de Sargan. La valeur associée à ce test indique qu'il n'y a pas de corrélation

Encadré 2

\section{LA MÉTHODE FGS2SLS}

Cette méthode d'estimation se déroule en trois étapes

1. Nous estimons tout d'abord un modèle autorégressif spatial par les doubles moindres carrés (IV-SAR) faisant appel aux variables instrumentales pour la variable endogène retardée spatialement (logarithme du prix du logement noté WLNPRIX) et pour la variable explicative endogène (le nombre de pièces noté Nbr_Pieces). Les variables exogènes sont introduites comme instruments. De plus, nous avons introduit les caractéristiques des acheteurs comme instruments supplémentaires (professions et catégories socioprofessionnelles, situation familiale, originaire ou non de la ville d'Angers). La liste des caractéristiques des acheteurs ainsi que leur statistique sont fournies en annexe 4

2. Les résidus obtenus à la première étape permettent ainsi une seconde estimation du paramètre $\lambda$ d'autorégression spatiale des erreurs, par la méthode des moments généralisés de Kelejian et Prucha (1999).

3. Enfin, nous ré-estimons par la méthode des doubles moindres carrés le modèle spécifié à la première étape dont les variables ont été reformulées selon une transformation spatiale de Cochrane-Orcutt (1949). Chaque variable est multipliée par le terme $(I-\hat{\lambda} M)$ où $\hat{\lambda}$ est le paramètre estimé lors de la seconde étape, I étant une matrice identité et $\mathrm{M}$ une matrice de poids.

La dépendance spatiale est prise en compte par le biais de cette matrice de poids. Les éléments diagonaux de cette dernière sont égaux à 0 tandis que les éléments non-diagonaux indiquent comment l'observation i est spatialement liée aux autres observations j. Cette matrice est définie par le modélisateur. Comme il n'existe pas de raisons a priori qu'une structure spatiale soit meilleure qu'une autre, plusieurs structures doivent être testées. Nous optons pour une structure du plus proche voisin avec des tests portant sur des nombres de voisins plus ou moins grands. En effet, le test portant sur d'autres structures de la matrice de pondération spatiale (structure de l'inverse de la distance notamment) ne maximisait pas l'indicateur global de degré d'autocorrélation spatiale entre nos unités spatiales. Ainsi, notre choix s'est porté sur une matrice de pondération des quatre plus proches voisins (cf. tableau ci-dessous), matrice maximisant l'indice de Moran. D'autre part, dans le cadre de notre transformation de Cochrane-Orcutt, nous avons utilisé une matrice de contiguïté avec une valeur seuil de 300 mètres afin d'optimiser nos estimations. 
significative entre les instruments et le terme d'erreur. Le test d'Hausman rejette quant à lui l'hypothèse nulle d'exogéneité du nombre de pièces. De plus, le test de nullité de l'indice de IV-Moran de Anselin et Kelejian (1997) ne rejette pas au seuil de $5 \%$ l'absence d'autocorrélation spatiale résiduelle dans le cadre de l'existence de variables endogènes. Ainsi la présence d'autocorrélation spatiale de même que l'endogénéité du nombre de pièces justifient le recours au modèle FGS2SLS. Le coefficient de la variable endogène décalée n'est pas significatif, ce qui traduit l'absence d'effets de débordement localisés sur le marché des appartements angevins pour les années 2004 et 2005.

Nos résultats confirment l'existence d'une autocorrélation spatiale positive et significative des erreurs $(\hat{\lambda}=0,164)$ : elle peut être analysée comme un effet de diffusion spatiale positive entre les prix immobiliers (Baumont, 2009). Cet effet est proche de celui obtenu par Baumont

Encadré 2 (suite)

Indice de Moran

\begin{tabular}{|l|l|}
\hline & \multicolumn{1}{|c|}{ Indice de Moran } \\
\hline 4 voisins & $0,38^{\star \star \star}$ \\
\hline 6 voisins & $0,36^{\star \star \star}$ \\
\hline 8 voisins & $0,35^{\star \star \star}$ \\
\hline 10 voisins & $0,33^{\star \star \star}$ \\
\hline 12 voisins & $0,33^{\star \star \star}$ \\
\hline 16 voisins & $0,31^{\star \star \star}$ \\
\hline 20 voisins & $0,28^{\star \star \star}$ \\
\hline 50 voisins & $0,19^{\star \star \star}$ \\
\hline
\end{tabular}

Lecture : les nombres de 4 à 50 correspondent au nombre de voisins.

*** : ce sigle indique que l'hypothèse nulle d'absence d'autocorrélation spatiale est rejetée au seuil de $1 \%$. Plus la valeur de l'indice de Moran est élevée, plus l'autocorrélation spatiale est importante.

Champ : échantillon de 1016 appartements vendus dans la ville d'Angers en 2004 et 2005.

Source : calculs des auteurs réalisés à partir de la base de données résultant de la fusion de la base PERVAL, des caractéristiques extrinsèques calculées à l'aide des logiciels FRAGSTATS et ArcGIS et des caractéristiques socio-économiques des IRIS fournies parl'Insee.

L'introduction de l'autocorrélation spatiale dans la fonction de prix hédoniques rendant difficile l'utilisation de la procédure Box-Cox pour le choix de la forme fonctionnelle, nous choisissons d'estimer les formes linéaire, semi-log (log-linéaire, linéaire-log) et double log. De même, seules les caractéristiques socioéconomiques des IRIS non corrélées avec les autres caractéristiques de l'appartement ont été conservées lors des estimations (cf. tableau 5). La part des logements de type HLM dans I'IRIS est, par exemple, corrélée positivement ou négativement avec la plupart des autres variables d'environnement socio-économique (par exemple, taux de chômage, personnes âgées de moins de 17 ans, etc.) et avec la densité des espaces verts autour de l'appartement. De même, le taux d'immigrés est corrélé avec le taux de chômage des 15-64 ans (cf. annexe 5). Par conséquent, il n'était donc pas économétriquement possible de conserver dans le modèle final l'ensemble de ces variables socio-économiques. Étant donné que l'article ne s'appuie pas, concernant ces variables d'environnement socio-économique, sur un modèle préalable hiérarchisant a priori les liens de causalité entre ces dernières, la sélection des variables socio-économiques retenues dans le modèle présenté s'est faite selon des considérations économétriques : parmi cet ensemble de variables d'environnement socio-économique, nous avons retenu celles qui conduisent au meilleur modèle économétrique selon la procédure stepwise, à savoir les ratios du taux de chômage des 15-24 ans, du taux d'appartement, du taux de personnes de plus de 65 ans et du taux d'établissements et d'entreprises.

La forme polytomisée de la variable correspondant au nombre d'étages a été également testée. Cependant, la valeur du test de Fisher étant inférieure à la valeur théorique, I'hypothèse de nullité des coefficients associés aux indicatrices de niveau a été acceptée. Nous n'avons donc pas retenu la forme polytomisée pour cette variable lors de nos estimations.

Afin de simplifier la présentation des résultats, nous ne présenterons par la suite que le modèle ayant le meilleur résultat en termes d'estimation: le critère utilisé pour effectuer ce choix est le coefficient de détermination $\left(R^{2}\right)$ ajusté. La forme fonctionnelle retenue est la forme double-log, les transformations logarithmiques étant appliquées au prix de l'appartement, aux différentes distances et à la surface de l'espace vert le plus proche. 
(2009) dans le cas de la ville de Dijon, ville dont les caractéristiques sont comparables à celles
d'Angers. Cet auteur observe, en effet, des valeurs allant de 0,22 à 0,23 selon le modèle retenu.

\begin{tabular}{|c|c|c|c|c|c|c|c|}
\hline \multicolumn{8}{|l|}{$\begin{array}{l}\text { Tableau } 5 \\
\text { Modèle hédonique }\end{array}$} \\
\hline \multirow[b]{2}{*}{ Constante } & \multicolumn{2}{|c|}{ MCO } & \multicolumn{2}{|c|}{ IV SAR } & \multicolumn{2}{|c|}{ FGS2SLS } & \multirow{2}{*}{$\begin{array}{c}\text { Élasticité } \\
\text { au point } \\
\text { moyen }\end{array}$} \\
\hline & $-1,444$ & & $-0,457$ & & $-0,002$ & & \\
\hline WLNPRIX & & & $-0,364$ & & $-0,421$ & & \\
\hline Nbr_piece & 0,302 & 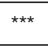 & 0,412 & 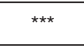 & 0,399 & 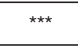 & 0,938 \\
\hline Étage & $-0,005$ & & $-0,006$ & & $-0,100$ & & $-0,194$ \\
\hline Sdb2_plus & 0,095 & & $-0,137$ & & $-0,485$ & $* \star \star *$ & $-0,010$ \\
\hline Anc_av_1850 & $-0,508$ & 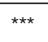 & $-0,489$ & 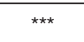 & $-0,567$ & 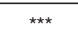 & $-0,008$ \\
\hline Anc_1850_1913 & $-0,505$ & $* \star *$ & $-0,571$ & $* * \star$ & $-0,520$ & *** & $-0,016$ \\
\hline Anc_1914_1947 & $-0,468$ & $* \star *$ & $-0,526$ & *** & $-0,529$ & $* \star *$ & $-0,023$ \\
\hline Anc_1948_1969 & $-0,443$ & 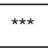 & $-0,537$ & *** & $-0,384$ & *** & $-0,069$ \\
\hline Anc_1970_1980 & $-0,342$ & 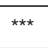 & $-0,392$ & $* \star \star \star$ & $-0,396$ & 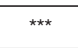 & $-0,051$ \\
\hline Anc_1981_1991 & $-0,390$ & 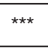 & $-0,383$ & 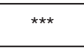 & $-0,048$ & 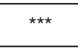 & $-0,003$ \\
\hline Anc_ap_1992 (Référence) & --- & 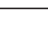 & --- & & -- & & -- \\
\hline Distance_Centre_ville & $-0,067$ & $\star \star *$ & $-0,063$ & & $-0,052$ & & $-0,037$ \\
\hline Distance_Santé & $-0,042$ & 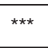 & $-0,086$ & & $-0,090$ & & $-0,064$ \\
\hline Distance_Axes_routiers & $-0,003$ & & $-0,020$ & & 0,162 & *夫 & 0,114 \\
\hline Distance_Cimetière & 0,018 & & 0,002 & & $-0,003$ & & $-0,002$ \\
\hline Distance_Vigne & 0,073 & & 0,080 & & $-0,005$ & & $-0,004$ \\
\hline Distance_Lande & 0,065 & & 0,094 & & 0,061 & & 0,043 \\
\hline Distance_Forêt & $-0,030$ & & $-0,015$ & & 0,093 & & 0,066 \\
\hline Distance_Esp_vert & $-0,041$ & 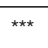 & $-0,049$ & ** & $-0,005$ & & $-0,004$ \\
\hline Densité_Esp_vert_1000m & 0,070 & 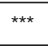 & 0,059 & ** & $-0,040$ & & $-0,868$ \\
\hline Superficie_Esp_vert & 0,003 & & 0,008 & & 0,072 & $* \star *$ & 0,051 \\
\hline Shannon_100m & 0,128 & 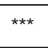 & 0,110 & *** & 0,012 & & 0,243 \\
\hline Agrégation_500m & 0,127 & $* \star \star *$ & 0,158 & * & 0,123 & $* \star *$ & 9,325 \\
\hline Taux_chômage_15_24ans & $-0,144$ & 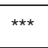 & $-0,150$ & 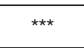 & $-0,158$ & *** & $-0,105$ \\
\hline Taux_Appartement & $-0,205$ & $* *$ & $-0,263$ & ** & $-0,222$ & ** & $-0,168$ \\
\hline Taux_Plus_65_ans & 0,116 & 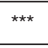 & 0,194 & ** & 0,159 & ** & 0,127 \\
\hline Taux_Entreprise_Établissement & $-0,022$ & 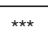 & $-0,016$ & & $-0,014$ & & $-0,107$ \\
\hline$\hat{\lambda}$ & & & & & 0,164 & ** & \\
\hline $\mathrm{R}^{2}$ ajusté & \multicolumn{2}{|c|}{0,67} & \multicolumn{2}{|c|}{0,63} & \multicolumn{2}{|c|}{0,66} & \\
\hline $\begin{array}{l}R^{2} 1 \text { ère étape } \\
\text { (WLNPRIX / Nbr_pieces) }\end{array}$ & & & \multicolumn{2}{|c|}{$0,24 / 0,27$} & \multicolumn{2}{|c|}{$0,64 / 0,28$} & \\
\hline Test de Pagan-Hall & & & 22,49 & 0,916 & & & \\
\hline Test de Sargan & & & 6,86 & 0,443 & 7,52 & 0,377 & \\
\hline Test d'Hausman & & & 5,11 & 0,006 & 9,88 & 0,007 & \\
\hline Test IV-Moran & & & 19,21 & 0,002 & & & \\
\hline
\end{tabular}

Lecture : ce tableau présente tout d'abord l'estimation de la fonction de prix hédoniques par la méthode des moindres carrés ordinaires. II présente ensuite les résultats obtenus pour le modèle IV-SAR (première étape de l'estimation par FGS2SLS) ainsi que ceux obtenus dans le cadre de l'estimation FGS2SLS (3 $3^{\text {ème }}$ étape). Les variables de ce dernier modèle sont les variables transformées selon la procédure de Cochrane-Orcutt. Pour chacune des variables explicatives, sont indiquées la valeur du coefficient estimé par ces différentes méthodes ainsi que leur significativité. Les valeurs du coefficient de détermination ajusté sont également présentées pour chaque modèle. Pour le modèle FGS2SLS, l'élasticité a été calculée au point moyen pour chacune des variables explicatives, cette dernière incluant l'effet spatial. Sont également présentés les différents tests associés à ces estimations. Enfin, les valeurs obtenues pour les Facteurs d'Inflation de la Variance de chacune des variables explicatives indiquent qu'il n'existe pas de problème de multicolinéarité entre ces dernières (VIF <6).

Les astérisques ***, **, * indiquent respectivement une significativité aux seuils de $1 \%, 5 \%$ et $10 \%$.

Champ : échantillon de 1016 appartements vendus dans la ville d'Angers en 2004 et 2005.

Source : calculs des auteurs réalisés à partir de la base de données résultant de la fusion de la base PERVAL, des caractéristiques extrinsèques calculées à l'aide des logiciels FRAGSTATS et ArcGIS et des caractéristiques socio-économiques des IRIS fournies par I'Insee. 


\section{La taille et l'ancienneté s'avèrent être des caractéristiques intrinsèques influentes}

L'analyse de la fonction de prix hédoniques estimée par la méthode FGS2SLS permet tout d'abord de mettre en évidence l'importance de certaines caractéristiques intrinsèques des appartements dans le choix résidentiel des ménages angevins. Ainsi, le fait de passer d'un appartement de trois pièces à quatre pièces conduit à une augmentation du prix de vente de $34,4 \%{ }^{18}$. Nous remarquons également qu'un appartement construit avant 1992 a, toutes choses égales par ailleurs, un prix plus bas que ceux construits après cette date. Les logements récents sont ainsi davantage valorisés par les ménages, résultat également obtenu par Cavailhès (2005).

\section{L'influence notable des espaces verts sur le prix des logements tient à leur plus ou moins grande superficie}

Le modèle final permet également de mettre en lumière un ensemble de caractéristiques extrinsèques ${ }^{19}$ des appartements ayant une influence significative sur le prix de ces logements. Ces caractéristiques étant par essence des éléments spatialisés, la mise en œuvre du modèle FGS2SLS permettant de traiter simultanément les questions de l'endogénéité et de l'autocorrélation spatiale peut conduire pour ces variables à des résultats différents de ceux issus de la mise en œuvre d'un simple modèle de MCO.

Ainsi, concernant le rôle du centre-ville d'Angers, le modèle des MCO montre un effet négatif et significatif de la distance, indiquant, si l'on suit ce modèle, une valorisation du centreville par les ménages résidents, ce qui irait dans le sens des résultats obtenus par Ahamada et al. (2007) pour le marché immobilier brestois. Cependant, ce résultat est biaisé pour les raisons évoquées en amont; la mise en œuvre du modèle FGS2SLS, si elle change peu la valeur du coefficient associé à cette variable $(-0,052$ au lieu de -0,067), indique que la distance par rapport au centre-ville n'est plus significative. On peut tenter d'expliquer ce résultat par le fait qu'introduire dans un modèle hédonique une variable de centre-ville consiste de fait à agréger en un seul «bouquet » l'ensemble des éléments qui composent le centre urbain (théâtre, château, restaurants, façades des anciennes maisons, etc.) et donc à considérer que ces derniers se localisent en un même point géographique, alors que dans la réalité ces éléments se dispersent sur une zone géographique centrale plus ou moins étendue selon la ville considérée. Cependant, la méthode consistant à désagréger la variable de centre-ville entre ses éléments composants, en dehors du fait qu'elle nécessite de les localiser géographiquement et de calculer les distances pour chacun d'entre eux, engendre une difficulté supplémentaire : la multicolinéarité entre ces différentes variables de centre-ville.

C'est ce type de difficulté qui a été rencontré lorsque nous avons opté pour l'introduction dans le modèle économétrique d'un ensemble d'indicateurs caractérisant l'environnement socio-économique du logement (cf. encadré 2). Nombre de ces caractéristiques sociodémographiques étant corrélées, la plupart n'apparaissent pas dans le modèle économétrique, sans pour autant que cela signifie qu'elles ne jouent pas sur les choix résidentiels des ménages. Ainsi, l'importance de l'environnement sociodémographique sur le prix des logements n'apparaît dans nos estimations qu'au travers de trois variables, à savoir la surreprésentation du taux de chômage des jeunes, la surreprésentation du nombre d'appartements (agissant toutes deux de manière négative sur les prix) et la surreprésentation de la population âgée de plus de 60 ans (agissant de manière positive sur les prix), ce dernier élément allant dans le sens des résultats obtenus par Baumont et Legros (2009) qui indiquent une élasticité de l'ordre de 0,12 pour la métropole parisienne en 1999 .

Par ailleurs, la proximité d'un logement par rapport à un axe routier majeur (autoroute, rocade, etc.) agit négativement et de manière significative sur le prix, avec une élasticité du prix par rapport à cette distance de l'ordre de 0,11 au niveau moyen de l'échantillon. Les nuisances associées à ces infrastructures routières l'emportent sur les avantages qu'elles apportent en termes d'accessibilité. Une fois de plus, les résultats issus du modèle des MCO divergent de

18. Par contre, la possession d'un appartement doté d'au moins deux salles de bain a un effet négatif en faisant baisser, dans le modèle FGS2LS, le prix du logement de $27 \%$, ce qui contraste Les calculs ont été refaits avec différentes matrices de poids et conduisent au même résultat. L'explication de ce résultat a priori contre-intuitif réside peut-être dans le fait que le nombre d'appartement dotés d'au moins deux salles de bain est très faible $(3,5 \%$ de l'échantillon) et se concentre pour plus de la moitié d'entre eux dans le quartier du Ralliement, caractérisé par de très vieux appartements.

19. Pour une caractéristique mesurée en termes de distance (ex. Distance_Forêt), un signe négatif du coefficient associé signifie que cette caractéristique est appréhendée comme une aménité, puisqu'un allongement de la distance engendre une baisse du prix du logement. 
ceux issus du modèle FGS2SLS : en effet, dans le premier modèle, cette distance aux infrastructures routières n'a pas d'impact significatif sur le prix des appartements.

Ces divergences entre les deux modèles économétriques apparaissent également lorsqu'on se focalise sur le rôle des aménités « vertes » : alors que le modèle des $\mathrm{MCO}$ indique que les espaces verts agissent comme des aménités via la significativité des variables de distance et de densité, leur superficie n'agissant pas de manière significative, le modèle FGS2SLS indique que ce rôle d'aménité s'exerce uniquement au travers de leur superficie : au niveau moyen de l'échantillon, une hausse de $1 \%$ de cette superficie conduirait donc à une hausse du prix du logement de $0,051 \%$, constat qui converge avec celui de Kong et al. (2007) concernant l'impact de la superficie des espaces verts sur le prix du logement (Jinan, Chine). Ce résultat associé au modèle FGS2SLS conduit à affirmer que les ménages considérés ont une préférence pour les grands espaces verts. Au travers de cette discussion sur le choix des modèles économétriques à mettre en œuvre, ce n'est donc pas tant le statut ou non d'aménité des espaces verts qui en jeu, mais bien la configuration de ces derniers qui importe pour les ménages.

Enfin, concernant l'occupation des sols, les résultats indiquent que les ménages angevins semblent préférer une agrégation des différents types d'occupation du sol dans un rayon de 500 mètres autour de leur appartement. Ce constat est cohérent avec la préférence des angevins pour de grands espaces verts en petit nombre (même si la question de l'occupation des sols ne se réduit pas uniquement à la question des espaces verts).

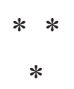

La mise en œuvre de la méthode des prix hédoniques permet donc de mettre en lumière le rôle de différents éléments de l'environnement urbain dans les choix de localisation des ménages. Basée sur des comportements observés, l'avantage de cette méthode est d'informer sans a priori sur le statut d'aménité ou de désaménité d'une caractéristique donnée, et ce, au travers du signe de son prix marginal. Cette neutralité se révèle être un avantage important lorsqu'il s'agit d'analyser sans parti-pris le rôle de certaines caractéristiques de l'environnement sociodémographique et économique du logement en vue d'aboutir à une compréhension fine des facteurs déterminant les choix résidentiels des ménages. Concernant le rôle des espaces verts en milieu urbain, la mise en œuvre de cette méthode permet également d'aller au-delà des évidences : si ces espaces verts sont apparus, conformément à l'intuition, comme des aménités agissant de manière significative sur les prix des appartements, le cas étudié met en relief le type de configuration souhaité par les ménages angevins, à savoir des espaces verts de grande taille.

En liaison avec la question de la mise en évidence des caractéristiques extrinsèques agissant de manière significative sur le prix des logements, cet article confirme l'importance du choix des modèles économétriques sur les résultats obtenus et les interprétations qui en découlent. Ainsi, la mise en œuvre d'un modèle de MCO sur une problématique de prix hédoniques portant sur le rôle des aménités (par nature spatialisées), peut conduire à des résultats biaisés voire à des interprétations opposées à celles obtenues par la mise en œuvre de modèles plus adaptés.

Cependant, du fait de la structure de la ville d'Angers, nous nous sommes focalisés uniquement sur le marché des appartements. Concernant les maisons individuelles, les résultats auraient été vraisemblablement différents, notamment concernant la valeur accordée par les ménages résidents aux espaces verts. La solution consistant à agréger le marché des appartements et celui des maisons individuelles ne nous est pas parue pertinente du fait de la nature foncièrement différente du choix entre ces deux types de biens immobiliers. Inversement, comparer les valeurs hédoniques obtenues pour chacun des deux marchés ne peut avoir qu'une portée limitée du fait d'une répartition spatiale singulièrement différente. La solution d'un modèle à deux étages consistant à coupler un modèle de choix discret (appartement versus maison individuelle) à un modèle de prix hédoniques reste à explorer. 


\section{BIBLIOGRAPHIE}

Ahamada I., Flachaire E. et Lubat M. (2007), «Prix des logements et autocorrélation spatiale: une approche semi-paramétrique », Économie Publique, $\mathrm{n}^{\circ}$ 20, pp. 131-145, Idep.

Alonso W. (1964), «Location and land use Towards a general theory of land rent », Harvard University press, Cambridge.

Anderson S.T. et West S.E. (2006), « Open space, residential property values, and spatial context», Regional Science and Urban Economics, $\mathrm{n}^{\circ} 36$, pp. 773-789.

Anselin L. et Kelejian H. H. (1997), « Testing for spatial error autocorrelation in the presence of endogenous regressors ", International Regional Science Review, vol. ${ }^{\circ} 20$, pp. 153-182.

Baumont C. et Legros D. (2009), « Neighborhood effects in spatial housing value models: The case of the metropolitan area of Paris (1999) » Document de travail du Laboratoire d'Économie et de Gestion, n e2009-09, Université de Bourgogne.

Baumont C. (2009), "Spatial effects of urban public policies on housing values ", Papers in Regional Science, vol. 88, $\mathrm{n}^{\circ}$ 2, pp. 301-326.

Bartik T.J. et Smith V.K. (1987), « Urban amenities and public policy » in Handbook of Regional and Urban Economics, E. S. Mills (ed.), vol. 2, chap. 31, pp. 1207-1254.

Blomquist G.C., Berger M.C. et Hoehn J. (1988), «New Estimates of Quality of Life in Urban Areas », American Economic Review, vol. $78, n^{\circ} 1$, pp. 89-107.

Bono P.H., Gravel N. et Trannoy A. (2007), «L'importance de la localisation dans la valorisation des quartiers marseillais ", Économie publique, $\mathrm{n}^{\circ} 20$, pp. 169-204, Idep.

Brueckner J.K., Thisse J.F. et Zenou Y. (1999), «Why is Central Paris Rich and Downtown Detroit Poor? An Amenity-Based Theory », European Economic Review, ${ }^{\circ}$ 43, pp. 91-107.

Can A. (1992), « Specification and Estimation of Hedonic Price Models », Regional Science and Urban Economics, ${ }^{\circ}$ 22, pp. 453-474.

Cavailhès J. (2005), « Le prix des attributs du logement », Économie et Statistique, n 381-382, pp. 91-123.
Cochrane D. et Orcutt G. H. (1949), «Application of least squares regression to relationships containing autocorrelated error terms », Journal of the American Statistical Association, vol. 44 , pp. $32-61$

Fingleton B. et Le Gallo J. (2008), « Estimating Spatial Models with Endogenous Variables, a Spatial Lag and Spatially Dependent Disturbances: Finite Sample Properties ", Papers in Regional Science, vol. 87, pp. 319-339.

Kelejian H. H. et Prucha I. R. (1998), «A Generalized Spatial Two-Stage Least Squares Procedure for Estimating a Spatial Autoregressive Model with Autoregressive Disturbances ", Journal of Real Estate Finance and Economics, vol. 17, n 1, pp. 99-121.

Kelejian H. H. et Prucha I. R. (1999), «A Generalized Moments Estimator for the Autoregressive Parameter in a Spatial Model», International Economic Review, vol. 40, $\mathrm{n}^{\circ} 2$, pp. 509-533.

Kim C.W., Phipps T.T. et Anselin L. (2003), «Measuring the benefits of air quality improvement: a spatial hedonic approach », Journal of Environmental Economics and Management, $\mathrm{n}^{\circ} 45$, pp. 24-39.

Kim K.S., Park S.J. et Kweon Y.J. (2007), « Highway Traffic Noise Effects on Land Price in an Urban Area », Transportation Research Part D, vol. $12, \mathrm{n}^{\circ} 4$, pp. 275-280.

Klaiber H.A. et Phaneuf D.J. (2010), « Valuing open space in a residential sorting model of the Twin Cities », Journal of Environmental Economics and Management, à paraître.

Kong F., Yin H. et Nakagoshi N. (2007), "Using GIS and landscape metrics in the hedonic price modelling of the amenity value of urban green space: A case study in Jinan City, China », Landscape and Urban Planning, $\mathrm{n}^{\circ} 79$, pp. 240-252.

Le Gallo J. (2002), «Économétrie spatiale: l'auto-corrélation spatiale dans les modèles de régression linéaire », Économie et Prévision, $\mathrm{n}^{\circ} 155$, pp. 139-158.

Le Gallo J. (2004), «Hétérogénéité spatiale, principes et méthodes ", Économie et Prévision, vol. 162, pp. 151-172. 
Mills E.S. (1967), «An aggregative model of resource allocation in a metropolitan area », American Economic Reviews, n ${ }^{\circ}$ 7, pp. 197-210.

Mills E.S. (1972), Studies in the Structure of the Urban Economy, Johns Hopkins Press, Baltimore.

Muth R.F. (1969), « Cities and housing: the spatial pattern of urban residential land use », The University of Chicago Press, Chicago.

Pagan A.R. et Hal D. (1983), « Diagnostic Tests as Residual Analysis », Econometric Reviews, vol. $2, n^{\circ} 2$, pp. 159-218.

Roback J. (1982), « Wages, rents, and the quality of life », Journal of Political Economy, $\mathrm{n}^{\circ}$ 90, pp. 1257-1278.

Rosen S. (1974), «Hedonic prices and implicit markets : product differentiation in pure competition », Journal of Political Economy, $\mathrm{n}^{\circ} 82$, pp. 34-55.

Rosen, S. (1979), «Wage-based indexes of urban quality of life » in Current Issues in Urban
Economics, Mieszkowski P., Straszheim M. (ed.), Johns Hopkins University Press, Baltimore.

Sander H.A., Polasky S. et Haight R.G. (2010), "The value of urban tree cover: A hedonic property price model in Ramsey and Dakota Counties, Minnesota, USA», Ecological Economics, vol. $69, \mathrm{n}^{\circ} 8$, pp. 1646-1656.

Sheppard S. (1987), « Hedonic analysis of housing markets ", in Handbook of regional and urban economics, Cheshire C.et Mills E. S (ed.), vol 3, chap. 41, pp. 1595-1635.

Straszheim M. (1987), «The Theory of Urban Residential Location », in Handbook of Regional and Urban Economics, E. S. Mills (ed.), vol. 2, chap. 18, pp. 717-757.

Travers M. (2007), Méthode des prix hédoniques et évaluation des actifs environnementaux : application au cas du littoral, Thèse de doctorat, Brest, 452 p.

Travers M., Nassiri A., Appere G. et Bonnieux F. (2008), «Évaluation des bénéfices environnementaux par la méthode des prix hédoniques: une application au cas du littoral », Économie et Prévision, $\mathrm{n}^{\circ} 185$, pp. 47-62. 
DÉCOMPOSITION EN IRIS DES QUARTIERS DE LA VILLE D'ANGERS

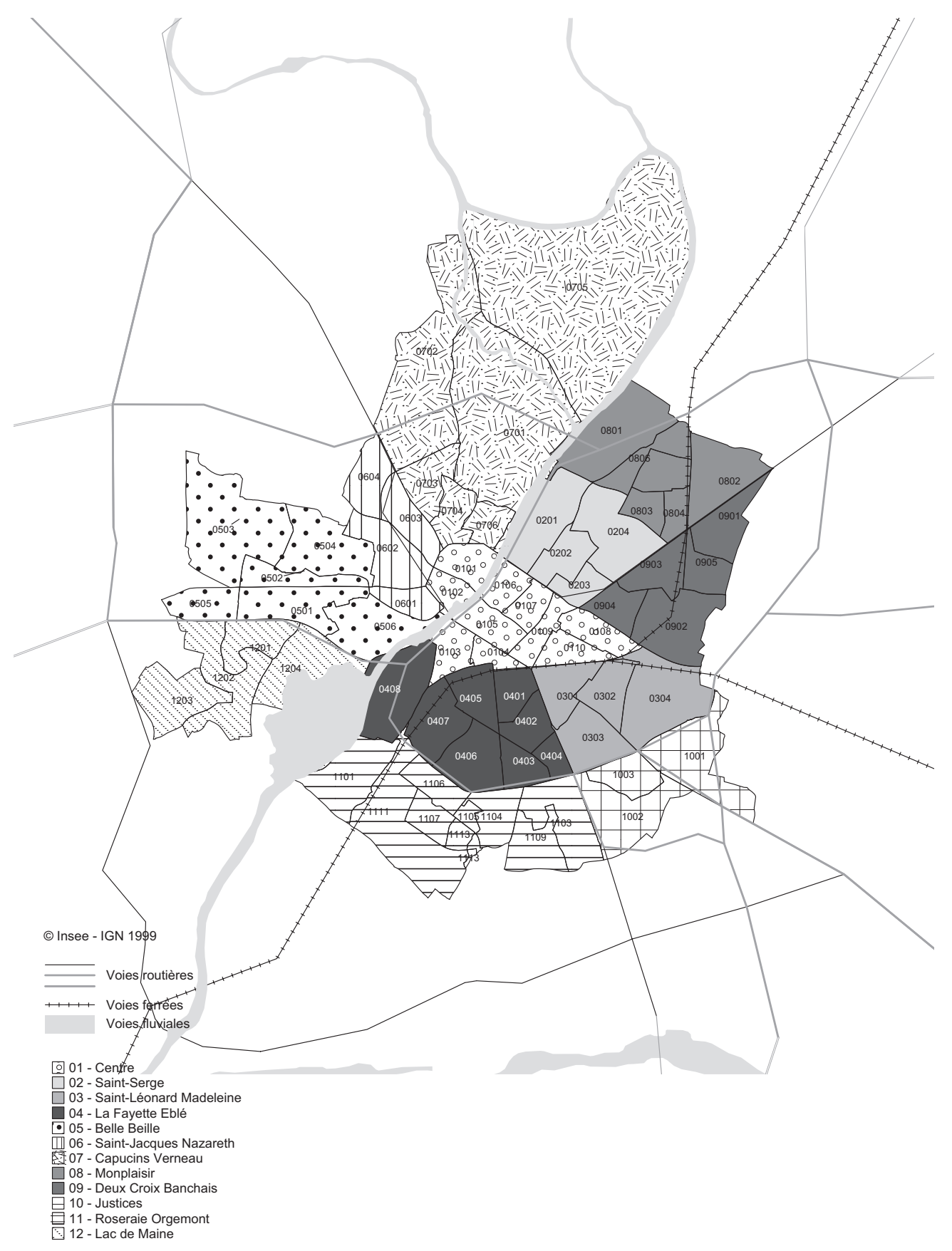

Lecture : Le nombre 0107 correspond à I'IRIS d'habitat n'0107. Rappelons que chaque IRIS est défini comme une zone homogène en termes d'indicateurs socio-démographiques et dont la population se situe entre 1800 et 5000 habitants. L'ensemble des IRIS dont les deux premiers chiffres de gauche sont identiques constitue un quartier de la commune d'Angers.

Champ : Commune d'Angers

Source : Insee-IGN 1999 
ANNEXE 2

\section{LES INDICATEURS UTILISÉS}

\begin{tabular}{|c|c|}
\hline Indicateur & Formule \\
\hline Superficie_Esp_vert & $\begin{array}{l}a_{j}: \text { aire de l'espace vert }\left(\mathrm{m}^{2}\right) \\
j \text { étant l'espace vert le plus proche de l'appartement }\end{array}$ \\
\hline Densité_Esp_vert_1000m & $\begin{array}{c}\text { Densité_Esp_vert }=\frac{\mathrm{n}}{\mathrm{A}} \times(10000) \times(100) \\
n: \text { nombre total d'espaces verts dans un rayon de } 1000 \text { mètres autour de l'appartement }\end{array}$ \\
\hline Agrégation_500m & $\begin{array}{l}\text { Agrégation }=\left[\sum_{i=1}^{m}\left(\frac{g_{i i}}{\max g_{i i}}\right) \times \mathrm{P}_{i}\right] \times 100 \\
\mathrm{~g}_{i i}: \text { nombre d'adjacences entre pixels de même classe } i, i \text { étant le type d'occupation du sol } \\
\text { max } \mathrm{g}_{\mathrm{ii}}: \text { nombre maximum d'adjacences potentielles entre pixels de même classe } i \\
\mathrm{P}_{i}: \text { proportion du sol occupé par la classe i dans un rayon de } 500 \text { mètres autour de l'appartement }\end{array}$ \\
\hline Shannon_100m & $\begin{array}{l}\text { Shannon }=-\sum_{i=1}^{m}\left(P_{i} \times \ln P_{i}\right) \\
P_{i} \text { : proportion du sol occupé par la classe i dans un rayon de } 100 \text { mètres autour de l'appartement }\end{array}$ \\
\hline
\end{tabular}

Lecture : à chaque indicateur est associée la formule ayant permis son calcul.

Champ : échantillon de 1016 appartements vendus dans la ville d'Angers en 2004 et 2005.

Source : d'après le logiciel FRAGSTATS. 
ANNEXE 3

STATISTIQUES DESCRIPTIVES DE L'ÉCHANTILLON

\begin{tabular}{|c|c|c|c|c|}
\hline Variables & Moyenne & Écart-type & Minimum & Maximum \\
\hline Prix_ttc $(€)$ & 107245,80 & 65420,27 & 18000,00 & 708504,30 \\
\hline Nbr_piece & 2,72 & 1,24 & 1 & 8,00 \\
\hline Sdb2_plus & 0,04 & 0,19 & 0 & 1 \\
\hline Étage & 2,75 & 2,88 & 0 & 15,00 \\
\hline Anc_av_1850 & 0,02 & 0,16 & 0 & 1 \\
\hline Anc_1850_1913 & 0,06 & 0,23 & 0 & 1 \\
\hline Anc_1914_1947 & 0,08 & 0,27 & 0 & 1 \\
\hline Anc_1948_1969 & 0,31 & 0,46 & 0 & 1 \\
\hline Anc_1970_1980 & 0,22 & 0,41 & 0 & 1 \\
\hline Anc_1981_1991 & 0,10 & 0,31 & 0 & 1 \\
\hline Anc_ap_1992 & 0,21 & 0,41 & 0 & 1 \\
\hline Distance_Centre_ville (m) & 1367,58 & 769,37 & 44,07 & 3954,44 \\
\hline Distance_Santé (m) & 689,38 & 365,64 & 13,09 & 1827,90 \\
\hline Distance_Axe_routiers $(\mathrm{m})$ & 886,47 & 383,08 & 79,36 & 1834,09 \\
\hline Distance_Cimetière $(\mathrm{m})$ & 1254,25 & 642,04 & 53,77 & 2741,35 \\
\hline Distance_Vigne $(\mathrm{m})$ & 4683,33 & 954,33 & 2535,51 & 7677,07 \\
\hline Distance_Lande $(\mathrm{m})$ & 2887,16 & 770,73 & 525,80 & 4929,14 \\
\hline Distance_Forêt (m) & 989,34 & 375,27 & 23,06 & 1712,82 \\
\hline Distance_Esp_vert (m) & 307,12 & 190,24 & 1,00 & 921,40 \\
\hline Densité_Esp_vert_1000m & 1,95 & 0,74 & 0,32 & 4,36 \\
\hline Superficie_Esp_vert $\left(\mathrm{m}^{2}\right)$ & 49270,34 & 87644,77 & 4100,82 & 573576,90 \\
\hline Shannon_100m & 0,39 & 0,40 & 0 & 1,32 \\
\hline Agrégation_500m & 97,41 & 0,75 & 95,56 & 99,14 \\
\hline Taux_chômage_15_24ans (\%) & 18,34 & 9,57 & 2,09 & 48,98 \\
\hline Taux_chômage_15_64ans (\%) & 14,58 & 6,30 & 5,84 & 38,16 \\
\hline Taux_ACC_15_64ans (\%) & 3,30 & 1,51 & 0,48 & 7,63 \\
\hline Taux_CPIS_15_64ans (\%) & 16,22 & 8,96 & 0,84 & 38,87 \\
\hline Taux_PI_15_64ans (\%) & 26,09 & 6,54 & 5,96 & 36,91 \\
\hline Taux_Emp_15_64ans (\%) & 30,88 & 5,40 & 16,24 & 42,10 \\
\hline Taux_Ouv_15_64ans (\%) & 22,22 & 9,99 & 7,59 & 50,72 \\
\hline Taux_0_17 ans (\%) & 19,39 & 6,70 & 7,07 & 37,12 \\
\hline Taux_Plus_65_ans (\%) & 15,16 & 5,82 & 2,27 & 31,60 \\
\hline Taux_Famille_3enfants_ou_plus (\%) & 5,28 & 3,84 & 0,98 & 17,08 \\
\hline Taux_Population_Immigrée (\%) & 6,12 & 0,04 & 0,02 & 0,20 \\
\hline Taux_Appartement (\%) & 74,80 & 22,87 & 0,37 & 100,00 \\
\hline Taux_d'HLM (\%) & 28,87 & 24,52 & 0,13 & 90,43 \\
\hline Taux_Logement_Vacant (\%) & 4,57 & 2,01 & 0,42 & 12,34 \\
\hline Taux_Entreprise_Etablissement (\%) & 1,614 & 1,70 & 0,34 & 10,53 \\
\hline
\end{tabular}

Lecture : les variables sont présentées dans le corps du texte. La moyenne, l'écart-type, les valeurs minimum et maximum sont calculés pour chacune d'entre elles.

Champ : échantillon de 1016 appartements vendus dans la ville d'Angers en 2004 et 2005.

Source : calculs des auteurs réalisés à partir de la base de données résultant de la fusion de la base PERVAL, des caractéristiques extrinsèques calculées à l'aide des logiciels FRAGSTATS et ArcGIS et des caractéristiques socio-économiques des IRIS fournies par l'Insee. 
ANNEXE 4

DÉFINITION ET STATISTIQUES DESCRIPTIVES DES VARIABLES INSTRUMENTALES

\begin{tabular}{|l|l|c|c|c|c|}
\hline \multicolumn{1}{|c|}{ Variable } & \multicolumn{1}{|c|}{ Précisions } & Moyenne & Écart- type & Minimum & Maximum \\
\hline A_PCS1 & $=1$ si l'acheteur est agriculteur exploitant, 0 sinon & 0,01 & 0,09 & 0 & 1 \\
\hline A_PCS2 & $\begin{array}{l}\text { = } 1 \text { si l'acheteur est artisan, commerçant ou chef d'entreprise, } \\
\text { s sinon }\end{array}$ & 0,07 & 0,25 & 0 & 1 \\
\hline A_PCS3 & $\begin{array}{l}=1 \text { si l'acheteur est cadre ou appartient aux professions } \\
\text { intellectuelles supérieures, 0 sinon } \\
\text { (référence) }\end{array}$ & 0,24 & 0,43 & 0 & 1 \\
\hline A_PCS4 & $\begin{array}{l}=1 \text { si l'acheteur appartient aux professions intermédiaires, } \\
\text { o sinon }\end{array}$ & 0,31 & 0,46 & 0 & 1 \\
\hline A_PCS5 & $=1$ si l'acheteur est employé, 0 sinon & 0,12 & 0,33 & 0 & 1 \\
\hline A_PCS6 & $=1$ si l'acheteur est ouvrier, 0 sinon & 0,06 & 0,24 & 0 & 1 \\
\hline A_PCS7 & $=1$ si l'acheteur est retraité, 0 sinon & 0,17 & 0,38 & 0 & 1 \\
\hline A_PCS8 & $=1$ si l'acheteur est sans activité ou étudiant, 0 sinon & 0,02 & 0,15 & 0 & 1 \\
\hline A_Seul & $=1$ si l'acheteur ne vit pas en couple, 0 sinon & 0,13 & 0,34 & 0 & 1 \\
\hline A_Angers & $\begin{array}{l}=1 \text { si l'acheteur était domicilié à Angers avant l'achat } \\
\text { de l'appartement, 0 sinon }\end{array}$ & 0,48 & 0,50 & 0 & 1 \\
\hline
\end{tabular}

Lecture : ce tableau présente le nom, la définition et la nature des instruments utilisés dans les équations auxiliaires. Ce tableau présente également la moyenne, l'écart-type, les valeurs minimum et maximum pour chacune d'entre elles.

Champ : échantillon de 1016 appartements vendus dans la ville d'Angers en 2004 et 2005.

Source : calculs des auteurs réalisés à partir de la base de données résultant de la fusion de la base PERVAL, des caractéristiques extrinsèques calculées à l'aide des logiciels FRAGSTATS et ArcGIS et des caractéristiques socio-économiques des IRIS fournies par l'Insee. 
ANNEXE 5

MATRICE DE CORRELATION DES CARACTERISTIQUES DES APPARTEMENTS

\begin{tabular}{|c|c|c|c|c|c|c|c|c|c|c|c|c|c|c|c|c|c|c|c|c|c|c|c|c|c|c|c|c|c|c|c|c|c|c|}
\hline 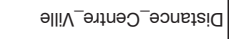 & & & & & & & & & & & & & & & & & & & & & & & & & & & & & & & & & a & \\
\hline 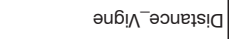 & & & & & & & & & & & & & & & & & & & & & & & & & & & & & & & & & $=$ & \\
\hline 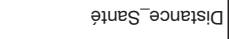 & & & & & & & & & & & & & & & & & & & & & & & & & & & & & & & & & : & \\
\hline 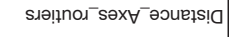 & & & & & & & & & & & & & & & & & & & & & & & & & & & & & & & & & 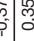 & \\
\hline әวə! $\mathrm{d}^{-}$גqN & & & & & & & & & & & & & & & & & & & & & & & & & & & & & & : & & & : & \\
\hline әрие ${ }^{-}$әэиетร! & & & & & & & & & & & & & & & & & & & & & & & & & & & & & 8 & 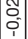 & $0^{\circ}$ & & $\begin{array}{lll}0 \\
0 \\
0 \\
0\end{array}$ & \\
\hline 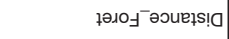 & & & & & & & & & & & & & & & & & & & & & & & & & & & & & & $\left|\begin{array}{lll}\mid & 2 \\
0 \\
0 \\
0 \\
0\end{array}\right|$ & ले & & (1) & \\
\hline 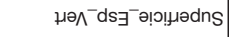 & & & & & & & & & & & & & & & & & & & & & & & & & & & & 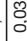 & 管 & $\left|\begin{array}{lll}0 \\
0 \\
0 \\
0\end{array}\right|$ & "1 & & $\begin{array}{c}n \\
n \\
n\end{array}$ & \\
\hline 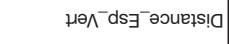 & & & & & & & & & & & & & & & & & & & & & & & & & & & $=\underbrace{}_{1}$ & $\begin{array}{l}0 \\
0 \\
i\end{array}$ & $\left.\mid \begin{array}{ll}0 \\
0 \\
0 \\
i\end{array}\right]$ & 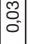 & 年 & & 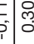 & 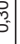 \\
\hline 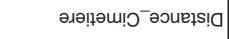 & & & & & & & & & & & & & & & & & & & & & & & & & & : & 党 & 心్ & 管 & $\left.\mid \begin{array}{l}2 \\
0 \\
0 \\
0 \\
1\end{array}\right]$ & 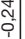 & & 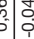 & \\
\hline $266 \mathrm{~L}^{-} \mathrm{de}-\mathrm{ou} \forall$ & & & & & & & & & & & & & & & & & & & & & & & & & & & 每 & $\int_{0}^{0}$ & on & $\begin{array}{ll}0 \\
0 \\
0 \\
0 \\
\end{array}$ & $\begin{array}{l}0 \\
0 \\
0 \\
\end{array}$ & & 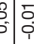 & \\
\hline 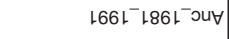 & & & & & & & & & & & & & & & & & & & & & & & & & $c_{1}^{\infty}$ & & $\int_{0}^{0}$ & & 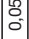 & {$\left[\begin{array}{ll}0 \\
0 \\
0 \\
0 \\
\end{array}\right]$} & s. & & $b^{\infty}$ & \\
\hline $086 \mathrm{~L}^{-} 0 \angle 6 \mathrm{~L}^{-}$ou $\forall$ & & & & & & & & & & & & & & & & & & & & & & & $\begin{array}{c}8 \\
- \\
-1\end{array}$ & & $\begin{array}{c}5 \\
\vdots \\
5\end{array}$ & 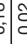 & 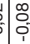 & $\frac{\infty}{i}$ & $\begin{array}{l}8 \\
0 \\
0\end{array}$ & $\begin{array}{l}5 \\
0 \\
0\end{array}$ & : & & Sִ & \\
\hline $696 \mathrm{~L}^{-} 8 t 6 \mathrm{~L}^{-}$ou $\forall$ & & & & & & & & & & & & & & & & & & & & & & & - l & 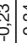 & 落. & & : & $\frac{\pi}{0}$ & 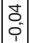 & $\mid$ & $\stackrel{9}{\circ}$ & ?. & $=0$ & \\
\hline$\angle \nabla 6 L^{-} \nabla L G L^{-}$ou $\forall$ & & & & & & & & & & & & & & & & & & & & & 욱 & ב్ & (1) & ?. & $\frac{1}{2}$ & s.t. & 突 & L & : & $\begin{array}{ll}1 \\
0 \\
0 \\
0\end{array}$ & בئ. & $\begin{array}{l}\sigma_{0}^{\circ} \\
0\end{array}$ & s.m & \\
\hline 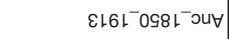 & & & & & & & & & & & & & & & & & & & & 8 & $\mid$ & $\begin{array}{c}0 \\
0 \\
0 \\
0\end{array}$ & $\begin{array}{c}n \\
0 \\
0\end{array}$ & - & vat & 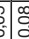 & g. & $\begin{array}{l}\infty \\
0 \\
0\end{array}$ & 要 & 覀 & $\frac{0}{1}$ & $\begin{array}{l} \\
0 \\
0\end{array}$ & \begin{tabular}{c}
3 \\
\hdashline \\
\end{tabular} & \\
\hline $0 S 8 L^{-} \Lambda E^{-} \mathrm{OUV}$ & & & & & & & & & & & & & & & & & & & 8 & $\mid \begin{array}{lll}0 \\
0 \\
0 \\
0\end{array}$ & $\left.\mid \begin{array}{l}0 \\
0 \\
0 \\
\vdots\end{array}\right]$ & $\begin{array}{c}F_{1} \\
\vdots\end{array}$ & 象 & : & 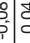 & & S. & $\frac{m}{0}$ & $\mid$ & $\mid$ & to & : & 5 & \\
\hline 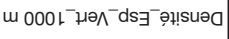 & & & & & & & & & & & & & & & & & & $\stackrel{8}{-}$ & $\mid \begin{array}{l}0 \\
0 \\
0\end{array}$ & : & $\left|\begin{array}{l}0 \\
0 \\
0 \\
\end{array}\right|$ & 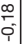 & 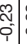 & : & 5 & s. & 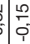 & $L_{0}^{\infty}$ & 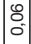 & 管 & 8 & & $\begin{array}{c}3 \\
0 \\
0\end{array}$ & \\
\hline m 00t'uouueus & & & & & & & & & & & & & & & & & 음 & : & 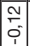 & \begin{tabular}{|l|l}
0 \\
0 \\
0
\end{tabular} & 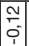 & $\begin{array}{l} \\
8 \\
0 \\
\end{array}$ & $\begin{array}{l} \\
0 \\
0\end{array}$ & 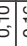 & $\frac{0}{5}$ & 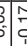 & $=\frac{0}{\circ}$ & $\begin{array}{l}2 \\
\text { N } \\
\text { in }\end{array}$ & $\begin{array}{l}7 \\
0\end{array}$ & 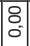 & $\frac{N}{2}$ & & s. & \\
\hline 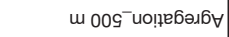 & & & & & & & & & & & & & & & & c & $\left\{\begin{array}{l}0 \\
8 \\
8\end{array}\right.$ & 象 & $\begin{array}{ll}1 \\
f_{0}\end{array}$ & 今. & {$\left[\begin{array}{l}n \\
0 \\
0 \\
0\end{array}\right]$} & $\begin{array}{ll}\sigma_{0} \\
0\end{array}$ & (1) & 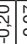 & bis & ic & : & $\mathscr{m}_{0}^{\infty}$ & $\begin{array}{ll}0 \\
0 \\
0 \\
0 \\
1\end{array}$ & : & 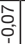 & & 年 & \\
\hline ә6ет & & & & & & & & & & & & & & & 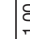 & $=1$ & $\bar{y}$ & $\frac{1}{0}$ & \begin{tabular}{|l|} 
\\
0 \\
0
\end{tabular} & $\begin{array}{c}5 \\
\\
\end{array}$ & 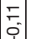 & 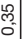 & $\begin{array}{l}0 \\
0 \\
0\end{array}$ & s. & 5 & $\infty$ & $\int_{0}^{2}$ & $\frac{7}{0}$ & \begin{tabular}{|l|l} 
\\
0 \\
0
\end{tabular} & : & $\frac{9}{0}$ & $\frac{1}{0}$ & 8 & \\
\hline sn।d ${ }^{-}$zqps & & & & & & & & & & & & & & & ;. & ba & $\bar{\delta}$ & : & : & i: & : & $\begin{array}{ll}\hat{0} \\
0 \\
0\end{array}$ & : & : & : & 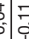 & $\bar{s}$ & o. & 훙 & 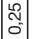 & $\bar{c}_{0}$ & $\begin{array}{lll}5 \\
0\end{array}$ & $\begin{array}{lll}5 \\
5 \\
0\end{array}$ & \\
\hline 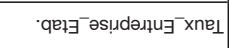 & & & & & & & & & & & & & & 8 & to & $\begin{array}{c}3 \\
3 \\
5\end{array}$ & ְ. & ? & $\begin{array}{l} \\
0 \\
0\end{array}$ & \begin{tabular}{|l|} 
\\
N \\
0
\end{tabular} & \begin{tabular}{|c|} 
\\
\\
0 \\
0
\end{tabular} & $\begin{array}{l} \\
\\
0 \\
0\end{array}$ & 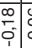 & 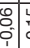 & 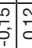 & 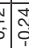 & : & W. & $\begin{array}{l}1 \\
0 \\
0 \\
i\end{array}$ & \begin{tabular}{|c|} 
\\
0 \\
0 \\
1
\end{tabular} & 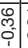 & $\begin{array}{c}8 \\
0\end{array}$ & $\begin{array}{lll}0 \\
5 \\
5\end{array}$ & \\
\hline$W 7 H_{4} P^{-} x n e_{\perp}$ & & & & & & & & & & & & & $\begin{array}{l}8 \\
- \\
-\end{array}$ & 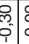 & कृ & 5 & $\sum_{i}^{\infty}$ & : & (F) & \begin{tabular}{|l|l} 
\\
0 \\
0
\end{tabular} & 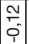 & 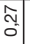 & $\begin{array}{ll}\infty \\
0 \\
0\end{array}$ & s. & 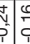 & : & 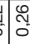 & $\frac{F}{5}$ & $\begin{array}{ll}1 \\
0 \\
0\end{array}$ & 吕. & $\bar{\delta}$ & 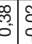 & 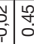 & \\
\hline ұиәшәдерdd $\forall^{-}$xne & & & & & & & & & & & & 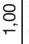 & $\begin{array}{l}\frac{1}{0} \\
\frac{1}{2}\end{array}$ & . & tis & bू & 票 & 0 & $\left|\begin{array}{l}n \\
0 \\
0 \\
0\end{array}\right|$ & $\begin{array}{ll}0 \\
0 \\
0\end{array}$ & $\mid \begin{array}{ll}m \\
0 \\
0 \\
\end{array}$ & $\begin{array}{ll}5 \\
0 \\
0 \\
0\end{array}$ & ז. & : & 象 & 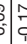 & 5 & స్ & $\begin{array}{ll}8 \\
0 \\
0\end{array}$ & 管 & 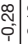 & $\begin{array}{l}0_{0} \\
0 \\
0\end{array}$ & 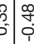 & \\
\hline ұиеэе $\wedge^{-}$ұ $^{-}$ & & & & & & & & & & & $\begin{array}{l}8 \\
\div \\
-\end{array}$ & $\begin{array}{l}y \\
0 \\
0\end{array}$ & בָּ & o. & ta & 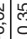 & $\frac{d}{\sqrt{n}}$ & 象 & $\begin{array}{ll}0 \\
0 \\
0\end{array}$ & $\frac{0}{0}$ & $\mid$ & $\begin{array}{ll} \\
0 \\
0\end{array}$ & $\begin{array}{l}\infty \\
\infty \\
\\
\end{array}$ & : & ta & 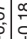 & : & ì & 象 & $\begin{array}{ll} \\
\\
0 \\
0\end{array}$ & $\frac{9}{0}$ & $\frac{1}{0}=\frac{5}{2}$ & $\frac{1}{2}$ & \\
\hline 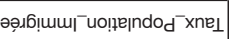 & & & & & & & & & & 家 & $\begin{array}{l}\text { sì } \\
0\end{array}$ & क्ष & $\left.\begin{array}{l}x_{0} \\
0 \\
0 \\
0\end{array}\right]$ & ס્ & bic & s. & กิ & 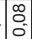 & \begin{tabular}{|l|l|}
$\begin{array}{l}0 \\
0 \\
0\end{array}$ \\
\end{tabular} & $\begin{array}{ll} \\
0 \\
0\end{array}$ & 合 & $\begin{array}{l} \\
0 \\
0\end{array}$ & ס. & 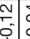 & tán & s. & $\frac{n}{6}$ & $0_{0}^{+}$ & $\begin{array}{c}1 \\
0 \\
0\end{array}$ & \begin{tabular}{|l|} 
\\
0 \\
0
\end{tabular} & $\begin{array}{l}\infty \\
\frac{\infty}{0} \\
\end{array}$ & $\begin{array}{c}0 \\
0 \\
0\end{array}$ & $\begin{array}{ll}0 \\
5 \\
5\end{array}$ & \\
\hline 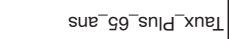 & & & & & & & & & \begin{tabular}{|c|} 
\\
-9
\end{tabular} & $\mid$ & $\begin{array}{ll}\bar{s} \\
0 \\
0\end{array}$ & $\begin{array}{l}8 \\
0 \\
0\end{array}$ & $F_{0}$ & c. & bू & a & $\sum_{0}^{\infty}$ & $\frac{m}{0}$ & $\mid \frac{\infty}{0}$ & $\frac{m}{0}$ & \begin{tabular}{|l|} 
\\
0 \\
0
\end{tabular} & 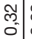 & 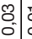 & 둥. & कृ & $\infty_{0}^{\infty}$ & $\bar{c}$ & $\frac{2}{0}$ & 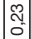 & \begin{tabular}{|l|l|} 
\\
0 \\
0 \\
0
\end{tabular} & $\begin{array}{c}0 \\
0 \\
0\end{array}$ & Oe & 5 & \\
\hline sue $\angle t^{-} 0^{-} \times n e_{\perp}$ & & & & & & & & : & $\begin{array}{l}2 \\
0 \\
0\end{array}$ & $\mid \begin{array}{l}0 \\
0 \\
0 \\
0\end{array}$ & $\begin{array}{c}-\overline{0} \\
0 \\
0\end{array}$ & $\begin{array}{cc}0 \\
0 \\
0\end{array}$ & 勇 & c. & : & à & $\frac{2}{0}$ & 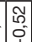 & s. & $\frac{2}{0}$ & $\begin{array}{l}\infty \\
0 \\
0 \\
\\
\end{array}$ & $\begin{array}{ll}\frac{\sigma}{0} \\
\sigma\end{array}$ & ר్. & 荡 & : & $\frac{5}{2}$ & $\frac{9}{0}$ & $\frac{\infty}{\infty}$ & $\begin{array}{ll}n \\
0 \\
0 \\
0\end{array}$ & $\mid$ & 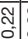 & \begin{tabular}{l|l} 
\\
and
\end{tabular} & 8 & . \\
\hline 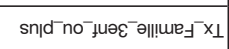 & & & & & & & 8 & $\begin{array}{l}\hat{\infty} \\
0 \\
0\end{array}$ & $\begin{array}{ll}0 \\
0 \\
0\end{array}$ & $\mid \begin{array}{l}\infty \\
0 \\
0 \\
0\end{array}$ & 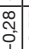 & $\begin{array}{ll}5 \\
\text { no } \\
0\end{array}$ & 商 & 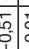 & s. & s: & $\bar{c}$ & $\begin{array}{l}\infty \\
0 \\
0 \\
0\end{array}$ & $\begin{array}{ll}2 \\
0 \\
0\end{array}$ & \begin{tabular}{|l|l|}
0 \\
\\
\end{tabular} & 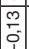 & $\begin{array}{l}0 \\
\\
0 \\
0\end{array}$ & స్తి & t: & : & : & 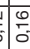 & $\frac{9}{5}$ & $\begin{array}{l}0 \\
0 \\
0\end{array}$ & th & $\frac{N}{0}$ & \begin{tabular}{l|l} 
\\
\\
\end{tabular} & 5 & |. \\
\hline sue $t 9^{-} \mathrm{Gl}^{-} \wedge \mathrm{nO}^{-} \mathrm{xne}$ & & & & & & s. & $\begin{array}{l}0 \\
0 \\
0 \\
0\end{array}$ & $\delta_{0}^{\circ}$ & 定 & 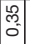 & 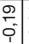 & $\begin{array}{ll}5 \\
0 \\
0\end{array}$ & 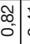 & की & ถึ. & 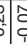 & on & $\begin{array}{l}0 \\
\\
\end{array}$ & 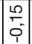 & $\begin{array}{ll}0 \\
0 \\
0 \\
1\end{array}$ & 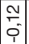 & 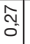 & ב⿳亠口冋. & 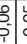 & 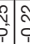 & s. & $\frac{9}{0}$ & స̃ & 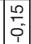 & 怼 & $\begin{array}{l}0 \\
0 \\
0\end{array}$ & F. & רִ & \\
\hline sue $t 9^{-}$st $^{-}$dum ${ }^{-} \times n e_{\perp}$ & & & & & 7 & $=\sqrt{0}$ & $\begin{array}{l}8 \\
0\end{array}$ & $\overbrace{0}^{\infty}$ & \begin{tabular}{|l|l} 
\\
0 \\
0
\end{tabular} & $\mid$ & $\begin{array}{ll} \\
0 \\
0\end{array}$ & 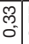 & $\begin{array}{l}\hat{N} \\
0 \\
0\end{array}$ & : & : & : & 旅 & $\mid \begin{array}{c}0 \\
0 \\
0 \\
0\end{array}$ & $\mid$ & $\begin{array}{l}8 \\
0 \\
0\end{array}$ & $\begin{array}{ll} \\
0 \\
0 \\
0\end{array}$ & $\begin{array}{ll} \\
\\
0 \\
0\end{array}$ & $\begin{array}{c}F_{0} \\
0\end{array}$ & 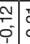 & 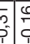 & 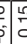 & $\overline{\bar{M}}$ & o: & 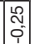 & F. & $\frac{0}{0}$ & $\frac{1}{2}$ & $\int_{5}^{\infty}$ & 5 \\
\hline sue $t 9^{-} G L^{-} l d^{-} x n e_{\perp}$ & & & & 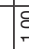 & 8 & : & $\begin{array}{l}\text { Nָ } \\
\text { S. }\end{array}$ & 웅 & $\left|\begin{array}{lll}0 \\
0 \\
0 \\
0 \\
0\end{array}\right|$ & $\mid$ & $\begin{array}{c}0 \\
0 \\
0 \\
0\end{array}$ & \begin{tabular}{l|l} 
\\
\\
0 \\
0
\end{tabular} & 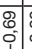 & : & ర్రి & ș & D্ & 柋 & : & 管 & $\mid \begin{array}{l}\mid \\
0 \\
0 \\
0\end{array}$ & 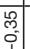 & $\begin{array}{l} \\
\\
0 \\
0\end{array}$ & 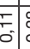 & : & tis & $\begin{array}{l}\infty \\
\\
0 \\
\end{array}$ & $\begin{array}{l}\text { to } \\
\end{array}$ & $\begin{array}{c}\approx \\
0 \\
0\end{array}$ & 年 & $\frac{m}{0}$ & t: & $\begin{array}{lll}0.0 \\
c\end{array}$ & \\
\hline sue $t 9^{-} \mathrm{GL}^{-}$SId ${ }^{-} \times \mathrm{Xne}_{\perp}$ & & & . & $=$ & (2) & 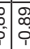 & 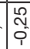 & 吕 & $\begin{array}{l}0 \\
0 \\
0 \\
1\end{array}$ & 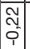 & $\bar{F}_{0}$ & $\begin{array}{l}0 \\
0 \\
0 \\
0\end{array}$ & 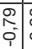 & ल్స్ & ס্: & 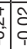 & $\frac{n}{6}$ & 5 & \begin{tabular}{|l|} 
\\
\\
0
\end{tabular} & \begin{tabular}{|l|l}
$\frac{1}{2}$ \\
0
\end{tabular} & \begin{tabular}{|l} 
\\
\\
$0^{\circ}$
\end{tabular} & 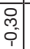 & $\begin{array}{l}\hat{n}_{0} \\
\bar{c}\end{array}$ & 8 & ș. & 5 & : & 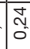 & \begin{tabular}{|l|l} 
\\
Na \\
0
\end{tabular} & 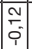 & 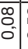 & \begin{tabular}{l|l}
0 \\
0 \\
0.0
\end{tabular} & $\begin{array}{ll}3 \\
\vdots \\
1 \\
1\end{array}$ & \\
\hline 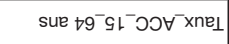 & & & $\begin{array}{l}8 \\
-\infty \\
c\end{array}$ & 象 & . & sin & & 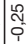 & \begin{tabular}{|l|l} 
\\
0 \\
0
\end{tabular} & $\mid \begin{array}{l}1 \\
0 \\
0 \\
\vdots\end{array}$ & 常 & 学 & 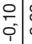 & 侦 & : & : & 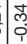 & $\frac{0}{0}$ & $\mid \begin{array}{ll}1 \\
0 \\
0\end{array}$ & $\begin{array}{l}m \\
0 \\
0\end{array}$ & $\mid \begin{array}{ll}\infty \\
0 \\
0 \\
0\end{array}$ & $\begin{array}{ll}\tilde{n} \\
0 \\
0\end{array}$ & 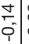 & $\begin{array}{l}8 \\
\\
\end{array}$ & 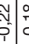 & 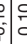 & 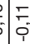 & 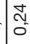 & 웅 & 立 & \begin{tabular}{ll}
\multicolumn{1}{c}{0} \\
$\circ$
\end{tabular} & : & 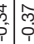 & \\
\hline 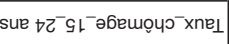 & & : & 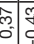 & 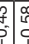 & 售. & $\frac{1}{2}$ & $\frac{2}{0}$ & $\prod_{0}^{\infty}$ & \begin{tabular}{|l|l} 
\\
0 \\
0
\end{tabular} & | & 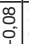 & 年 & 足 & ¿ิ & 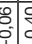 & tִ & 赵 & 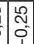 & $\begin{array}{ll}0 \\
0 \\
0 \\
0\end{array}$ & $\begin{array}{ll}m \\
0 \\
0\end{array}$ & 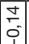 & | & ¿. & 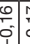 & $\hat{a}$ & 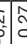 & $\frac{0}{0}$ & $\stackrel{5}{0}$ & $\begin{array}{ll}0 \\
0 \\
0 \\
0\end{array}$ & $\mid$ & ב. & : & 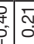 & \\
\hline \multirow[t]{2}{*}{ 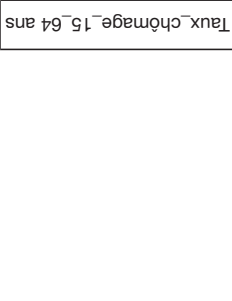 } & $\begin{array}{l}0 \\
-\end{array}$ & 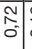 & $\begin{array}{l}\frac{9}{5} \\
\end{array}$ & רִ & | & 家 & $\frac{\infty}{0}$ & 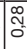 & $\begin{array}{l}\infty \\
\\
0\end{array}$ & \begin{tabular}{|c|} 
\\
0 \\
0
\end{tabular} & $\begin{array}{l}0 \\
\\
0\end{array}$ & 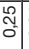 & 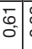 & 总 & $\begin{array}{l}0 \\
1 \\
\end{array}$ & $\begin{array}{ccc}\infty \\
c\end{array}$ & $\bar{m}$ & 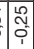 & \begin{tabular}{|l|} 
\\
0 \\
0 \\
\end{tabular} & \begin{tabular}{|c|}
0 \\
0 \\
0
\end{tabular} & \begin{tabular}{|l|} 
\\
0 \\
0
\end{tabular} & 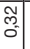 & ס्. & $\begin{array}{l}0 \\
0 \\
9 \\
\end{array}$ & $\begin{array}{l}3 \\
0 \\
0 \\
0\end{array}$ & s. & ñ & $\stackrel{N}{\circ}$ & $\begin{array}{l}1 \\
\overline{1} \\
\vdots\end{array}$ & $\begin{array}{l} \\
0 \\
0\end{array}$ & $\bar{\sigma}$ & 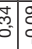 & $\begin{array}{lll}0 \\
\\
\end{array}$ & \\
\hline & 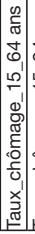 & 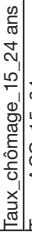 & 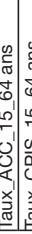 & 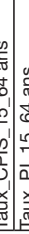 & 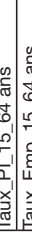 & 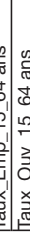 & 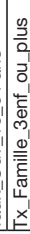 & 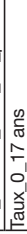 & 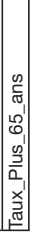 & 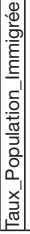 & 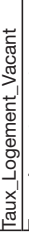 & 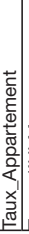 & 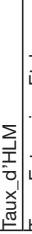 & 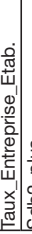 & $\begin{array}{l}\frac{2}{2} \\
\text { ר) } \\
0 \\
0\end{array}$ & & 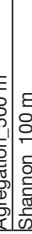 & 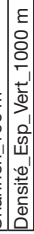 & 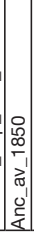 & \begin{tabular}{|c|} 
\\
$\frac{m}{\sigma}$ \\
$\frac{0}{0}$ \\
0 \\
0 \\
0 \\
0 \\
0 \\
0 \\
\end{tabular} & 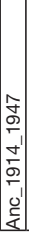 & 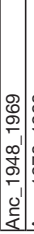 & 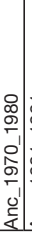 & 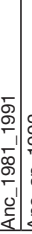 & 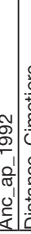 & 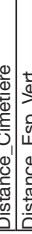 & 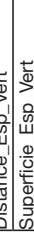 & 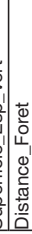 & 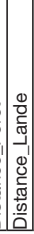 & 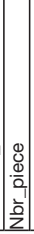 & & 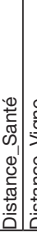 & 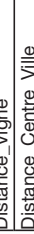 & \\
\hline
\end{tabular}


